\title{
Derived equivalences of gentle algebras via Fukaya categories
}

\author{
Yankı Lekili ${ }^{1}$ - Alexander Polishchuk ${ }^{2,3,4}$
}

Received: 8 February 2018 / Revised: 12 July 2019 / Published online: 29 August 2019

(c) The Author(s) 2019

\begin{abstract}
Following the approach of Haiden-Katzarkov-Kontsevich (Publ Math Inst Hautes Études Sci 126:247-318, 2017), to any homologically smooth $\mathbb{Z}$-graded gentle algebra $A$ we associate a triple $\left(\Sigma_{A}, \Lambda_{A} ; \eta_{A}\right)$, where $\Sigma_{A}$ is an oriented smooth surface with non-empty boundary, $\Lambda_{A}$ is a set of stops on $\partial \Sigma_{A}$ and $\eta_{A}$ is a line field on $\Sigma_{A}$, such that the derived category of perfect dg-modules of $A$ is equivalent to the partially wrapped Fukaya category of $\left(\Sigma_{A}, \Lambda_{A} ; \eta_{A}\right)$. Modifying arguments of Johnson and Kawazumi, we classify the orbit decomposition of the action of the (symplectic) mapping class group of $\Sigma_{A}$ on the homotopy classes of line fields. As a result we obtain a sufficient criterion for homologically smooth graded gentle algebras to be derived equivalent. Our criterion uses numerical invariants generalizing those given by Avella-AlaminosGeiss in Avella et al. (J Pure Appl Algebra 212(1):228-243, 2008), as well as some other numerical invariants. As an application, we find many new cases when the AAGinvariants determine the derived Morita class. As another application, we establish some derived equivalences between the stacky nodal curves considered in Lekili and Polishchuk (J Topology 11:615-444, 2018)
\end{abstract}

\section{Introduction}

Given a Liouville manifold $(M, \omega=d \lambda)$, a rigorous definition of the compact Fukaya category, $\mathcal{F}(M)$, appears in the monograph [27]. This is a triangulated $A_{\infty}$-category linear over some base ring $\mathbb{K}$. Roughly speaking, the objects of $\mathcal{F}(M)$ are com-

Communicated by Jean-Yves Welschinger.

$凶 \quad$ Yankı Lekili

yanki.lekili@kcl.ac.uk

1 King's College London, London, UK

2 National Research University Higher School of Economics, Moscow, Russia

3 Korea Institute for Advanced Study, Seoul, South Korea

4 University of Oregon, Eugene, USA 
pact, exact, oriented Lagrangian submanifolds in $M$, equipped with spin structures (if char $\mathbb{K} \neq 2$ ). The orientations on each Lagrangian determine a $\mathbb{Z}_{2}$-grading on $\mathcal{F}(M)$, and the spin structures enter in orienting the moduli spaces of holomorphic polygons that enter into the definition of structure constants of the $A_{\infty}$ operations. It is often convenient to upgrade the $\mathbb{Z}_{2}$-grading on $\mathcal{F}(M)$ to a $\mathbb{Z}$-grading, which can be done under the additional assumption that $2 c_{1}(M)=0$ (see $[20,26]$ ). Under this assumption, one defines a notion of a grading structure on $M$, and correspondingly considers only graded Lagrangians as objects of $\mathcal{F}(M)$, which now becomes a $\mathbb{Z}$ graded category. We refer to [26] for these general notions. In this paper, we focus our attention to the case where $M=\Sigma$ is a punctured (real) 2-dimensional surface, equipped with an area form. A grading structure on $\Sigma$ can be concretely described as a homotopy class of a section $\eta$ of the projectivized tangent bundle of $\mathbb{P}(T \Sigma)$. Note that there is an effective $H^{1}(\Sigma)$ 's worth of choices (see Sect. 1). A Lagrangian can be graded if the winding number of $\eta$ along $L$ vanishes, and in such a situation a grading is a choice of a homotopy from the tangent lift $L \rightarrow T L \subset T \Sigma$ to $\eta_{\mid L}$ along $L$. These gradings extend in a straightforward manner to the wrapped Fukaya category $\mathcal{W}(\Sigma)$ which contains $\mathcal{F}(\Sigma)$ as a full subcategory, but also allows non-compact Lagrangians in $\Sigma$ and more generally, partially wrapped category $\mathcal{W}(\Sigma, \Lambda)$, as studied in [15, Sec. 2.1], where $\Sigma$ is a surface with boundary and $\Lambda$ is a collection of stops (i.e., marked points) on $\partial \Sigma$.

By a graded surface $(\Sigma, \Lambda ; \eta)$ we mean an oriented surface with boundary $\Sigma$, together with a set $\Lambda$ of marked points on the boundary and a line field $\eta$. Given two graded surfaces with stops, $\left(\Sigma_{i}, \Lambda_{i} ; \eta_{i}\right)$ for $i=1,2$, and a homeomorphism $\phi: \Sigma_{1} \rightarrow \Sigma_{2}$, such that $\phi\left(\Lambda_{1}\right)=\Lambda_{2}$, and $\phi_{*}\left(\eta_{1}\right)$ is homotopic to $\eta_{2}$ (we refer to such homeomorphisms as graded $)$, one gets an equivalence between the partially wrapped Fukaya categories $\mathcal{W}\left(\Sigma_{1}, \Lambda_{1} ; \eta_{1}\right)$ and $\mathcal{W}\left(\Sigma_{2}, \Lambda_{2} ; \eta_{2}\right)$. Thus, it is important to have a set of explicit computable invariants of a line field $\eta$ on a surface with boundary that determine the orbit of $\eta$ under the action of the mapping class group of $\Sigma$. Our first result (see Theorem 1.8) gives such invariants in terms of winding numbers of $\eta$. In the most interesting case when genus is $\geq 2$, the invariants consist of the winding numbers along all the boundary components, plus two more invariants, each taking values 0 and 1 . The first of them decides whether the line field $\eta$ is induced by a non-vanishing vector field, while the second is the Arf-invariant of a certain quadratic form over $\mathbb{Z}_{2}$. The cases of genus 1 and 0 are special due to the special nature of the corresponding mapping class groups. In the case of genus 1 there is a certain $\mathbb{Z}$-valued invariant in addition to the winding numbers along boundary components. Note that from the numerical invariants of Theorem 1.8 one can also recover the genus of the surface and the numbers of stops on the boundary components, so if these invariants match then the corresponding partially wrapped Fukaya categories are equivalent.

Next, we use this result to construct derived equivalences between gentle algebras, introduced by Assem and Skowrónski in [3]. This is a remarkable class algebras with monomial quadratic relations of special kind with a well understood structure of indecomposable modules. Furthermore, their derived categories of modules also enjoy many nice properties (see [11] and references therein). Avella-Alaminos and Geiss [7] gave a combinatorial definition of derived invariants of finite-dimensional gentle algebras, which form a collection of pairs of non-negative integers $(m, n)$ with 
multiplicities. We refer to these as $A A G$-invariants. It is known that these invariants do not completely determine the derived Morita class of a gentle algebra in general (for example, see [1]).

We consider $\mathbb{Z}$-graded gentle algebras and their perfect derived categories (the classical case corresponds to algebras concentrated in degree 0 ). For such an algebra $A$, we denote by $D(A)$ the perfect derived category of dg-modules over $A$ viewed as a dg-algebra with zero differential. The category $D(A)$ has a natural dg-enhancement which we take into account when talking about equivalences involving $D(A)$.

The connection between graded gentle algebras and Fukaya categories was established by Haiden, Katzarkov and Kontsevich [15] (cf. [10]): they constructed collections of formal generators in some partially wrapped Fukaya categories whose endomorphism algebras are graded gentle algebras. In Theorem 3.11 we give an inverse construction $^{1}$ : starting from a homologically smooth graded gentle algebra $A$ we construct a graded surface with stops $\left(\Sigma_{A}, \Lambda_{A} ; \eta_{A}\right)$ together with a set formal generators whose endomorphism algebra is isomorphic to $A$. This leads to an equivalence of the partially wrapped Fukaya category $\mathcal{W}(\Sigma, \Lambda)$ with the derived category $D(A)$. In addition, we generalize the combinatorial definition of AAG-invariants to possibly infinite-dimensional graded gentle algebras and show that they can be recovered from the winding numbers of $\eta_{A}$ along all boundary components.

Now recalling our numerical invariants of graded surfaces with stops from Theorem 1.8 we obtain a sufficient criterion for derived equivalence between homologically smooth graded gentle algebras. Namely, if we start with two such algebras $A$ and $A^{\prime}$ and find that the corresponding invariants from Theorem 1.8, determined by winding numbers of $\eta_{A}$ and $\eta_{A^{\prime}}$, coincide then we get a derived equivalence between $A$ and $A^{\prime}$. More precisely, the first step is to check that $A$ and $A^{\prime}$ have the same AAG-invariants. In the case of genus 0 , this suffices. For genus 1 , one has to compute a certain invariant with values in $\mathbb{Z}_{\geq 0}$, while for genus $>1$ one has to check that two invariants with values in $\{0,1\}$ match. Note that the genus can be computed from the AAG-invariants.

As an application, using the above approach we obtain a sufficient criterion for derived equivalence of homologically smooth graded gentle algebras given purely in terms of AAG-invariants (see Corollary 3.14). Using Koszul duality, we also get a sufficient criterion for derived equivalence of finite-dimensional gentle algebras with grading in degree 0 (see Corollary 3.16).

In a different direction, we construct derived equivalences between stacky nodal curves studied in [21]. Namely, these are either chains or rings of weighted projective lines glued to form stacky nodes, locally modelled by quotients $(x y=0) /(x, y) \sim$ $\left(\zeta^{k} x, \zeta y\right)$, where $\zeta^{r}=1$ and $k \in(\mathbb{Z} / r)^{*}$. In [21, Thm. B] we constructed an equivalence of the derived category of coherent sheaves on such a stacky curve with the partially wrapped Fukaya category of some graded surface with stops (this can be viewed as an instance of homological mirror symmetry). Thus, using Theorem 1.8 we get many nontrivial derived equivalences between our stacky curves. In the case of balanced nodes (those with $k=-1$ ) we recover the equivalences between tcnc curves from [28].

\footnotetext{
1 The existence of such construction is mentioned in [15].
} 


\section{Line fields on surfaces}

\subsection{Basics on line fields}

Let $\Sigma$ be an oriented smooth surface of genus $g(\Sigma)$ with non-empty boundary with connected components $\partial \Sigma=\bigsqcup_{i=1}^{d} \partial_{i} \Sigma$. The pure mapping class group of $\Sigma$ is

$$
\mathcal{M}(\Sigma)=\pi_{0}\left(\text { Homeo }^{+}(\Sigma, \partial \Sigma)\right)
$$

where $\mathrm{Homeo}^{+}(\Sigma, \partial \Sigma)$ is the space of orientation preserving homeomorphism of $\Sigma$ which are the identity pointwise on $\partial \Sigma$.

Definition 1.1 An (unoriented) line field $\eta$ on $\Sigma$ is a section of the projectivized tangent bundle $\mathbb{P}(T \Sigma)$. We denote by

$$
G(\Sigma)=\pi_{0}(\Gamma(\Sigma, \mathbb{P}(T \Sigma)))
$$

the set of homotopy classes of unoriented line fields.

A non-vanishing vector field gives a section of the tangent circle bundle $\mathbb{S} \Sigma$. Such a section induces a line field via the bundle map $\mathbb{S} \Sigma \rightarrow \mathbb{P}(T \Sigma)$ which is a fibrewise double covering. However, not all line fields come from non-vanishing vector fields: a section of $\mathbb{P}(T \Sigma)$ may not lift to a section of $\mathbb{S} \Sigma$ (in Lemma 1.4 below we will get a criterion for this).

The trivial circle fibration

$$
S^{1} \stackrel{\iota}{\rightarrow} \mathbb{P}(T \Sigma) \stackrel{p}{\rightarrow} \Sigma
$$

induces an exact sequence

$$
0 \rightarrow H^{1}(\Sigma) \stackrel{p^{*}}{\rightarrow} H^{1}(\mathbb{P}(T \Sigma)) \stackrel{\iota^{*}}{\rightarrow} H^{1}\left(S^{1}\right) \rightarrow 0
$$

(here and below, when the coefficient group is omitted it is assumed to be $\mathbb{Z}$ ). Note that the orientation on $\Sigma$ induces orientations on the tangent circles, so that the inclusion $\iota$ used in the above sequence is canonical up to homotopy.

We can think of line fields as trivializations of the circle fibration (1.1), in particular, the set $G(\Sigma)$ has a natural structure of a torsor over the group of homotopy classes of maps $\Sigma \rightarrow S^{1}$, i.e., with $H^{1}(\Sigma)$. We denote the corresponding action of $c \in H^{1}(\Sigma)$ on $G(\Sigma)$ by $\eta \mapsto \eta+c$.

Let us associate with a line field $\eta$ the class $[\eta] \in H^{1}(\mathbb{P}(T \Sigma))$, such that $\iota^{*}[\eta]\left(\left[S^{1}\right]\right)=1$, by taking the Poincaré-Lefschetz dual of the class of the image $[\eta(\Sigma)] \subset H_{2}(\mathbb{P}(T \Sigma), \partial \mathbb{P}(T \Sigma))$.

Lemma 1.2 The map $\eta \mapsto[\eta]$ gives an identification

$$
G(\Sigma)=\left(\iota^{*}\right)^{-1}(\zeta) \subset H^{1}(\mathbb{P}(T \Sigma)),
$$

where $\zeta \in H^{1}\left(S^{1}\right)$ is the generator which integrates to 1 along $S^{1}$. 
Proof The exact sequence (1.2) shows that set $\left(\iota^{*}\right)^{-1}(\zeta)$ is a torsor over $H^{1}(\Sigma)$. It is easy to check that the map $\eta \mapsto[\eta]$ is compatible with the $H^{1}(\Sigma)$-actions, i.e.,

$$
[\eta+c]=[\eta]+p^{*} c
$$

The assertion follows immediately from this.

The mapping class group $\mathcal{M}(\Sigma)$ acts on $G(\Sigma)$ on the right. Our goal in this section is to understand the orbit decomposition of $G(\Sigma)$ with respect to this action.

Given an immersed curve $\gamma: S^{1} \rightarrow \Sigma$, one can consider its tangent lift $\tilde{\gamma}: S^{1} \rightarrow$ $\mathbb{P}(T \Sigma)$ given by $(\gamma, T \gamma)$, where $T \gamma$ is the tangent space to the curve $\gamma$.

Definition 1.3 Given a line field $\eta$ and an immersed curve $\gamma$, define the winding number of $\gamma$ with respect to $\eta$ to be

$$
w_{\eta}(\gamma):=\langle[\eta],[\tilde{\gamma}]\rangle
$$

where $\langle\rangle:, H^{1}(\mathbb{P}(T \Sigma)) \times H_{1}(\mathbb{P}(T \Sigma)) \rightarrow \mathbb{Z}$ is the natural pairing.

The winding number $w_{\eta}(\gamma)$ with respect to $\eta$ only depends on the homotopy class of $\eta$ and the regular homotopy class of $\gamma$. From the definition we immediately get the following compatibility with the action of $H^{1}(\Sigma)$ :

$$
w_{\eta+c}(\gamma)=w_{\eta}(\gamma)+\langle c,[\gamma]\rangle
$$

Throughout, $\partial \Sigma$ is oriented with respect to the natural orientation as the boundary of $\Sigma$. In particular, $w_{\eta}\left(\partial \mathbb{D}^{2}\right)=2$ for the unique homotopy class of line fields on $\mathbb{D}^{2}$. For a boundary component $B \subset \partial \Sigma$ with the opposite orientation, we write $-B$. Then, we have $w_{\eta}(-B)=-w_{\eta}(B)$.

Every nonvanishing vector field $v$ on $\Sigma$ defines naturally a line field. In this way we get a map

$$
V(\Sigma) \rightarrow G(\Sigma): v \mapsto\langle v\rangle
$$

from the set of homotopy classes of nonvanishing vector fields $V(\Sigma)$. We can think of nonvanishing vector fields as trivializations of the tangent circle bundle, so $V(\Sigma)$ has a natural action of the group of homotopy classes of maps $\Sigma \rightarrow S^{1}$, i.e., of $H^{1}(\Sigma)$. It is easy to check that the above map is compatible with the $H^{1}(\Sigma)$-actions via the multiplication by 2 :

$$
\langle v+c\rangle=\langle v\rangle+2 c
$$

for $c \in H^{1}(\Sigma)$. Also, for any nonvanishing vector field $v$, the winding number of the corresponding line field $\langle v\rangle$ along an immersed curve $\gamma$ is related to the winding number of $v$ itself by

$$
w_{\langle v\rangle}(\gamma)=2 w_{v}(\gamma)
$$


Lemma 1.4 A line field $\eta$ comes from a vector field if an only if all of its winding numbers are even.

Proof The "only if" part is clear. Now let $\eta$ be a line field with even winding numbers and let $v$ be some nonvanishing vector field (it exists since $\Sigma$ is noncompact). Then $\eta=\langle v\rangle+c$ for some $c \in H^{1}(\Sigma)$ such that $\langle c,[\gamma]\rangle$ is even for every homology class $[\gamma]$. But this implies that $c=2 c^{\prime}$, so $\eta=\left\langle v+c^{\prime}\right\rangle$.

\subsection{Invariants under the action of the mapping class group}

Recall that $\partial_{i} \Sigma, i=1, \ldots, d$ are the components of the boundary of $\Sigma$. Given a line field $\eta$, the winding numbers

$$
w_{\eta}\left(\partial_{i} \Sigma\right) \text { for } i=1, \ldots d
$$

depend only on the homotopy class of $\eta$ and are invariant under the action of the mapping class group $\mathcal{M}(\Sigma)$. This gives us the first set of invariants of elements of $G(\Sigma)$.

To go further, we need to study the winding numbers along non-separating curves on $\Sigma$. As is well-known, the winding number invariants do not descend to a map from $H_{1}(\Sigma)$. Indeed, if $S \subset \Sigma$ is a compact subsurface with boundary $\partial S=\bigsqcup_{i=1}^{d} \partial_{i} S$, by Poincaré-Hopf index theorem (see [16, Ch. 3]), we have:

$$
\sum_{i=1}^{d} w_{\eta}\left(\partial_{i} S\right)=2 \chi(S)
$$

However, considering the reduction modulo 2 we still get a well-defined homomorphism (see [17]):

$$
\left[w_{\eta}\right]^{(2)}: H_{1}\left(\Sigma ; \mathbb{Z}_{2}\right) \rightarrow \mathbb{Z}_{2}
$$

i.e an element $H^{1}\left(\Sigma ; \mathbb{Z}_{2}\right)$.

Definition 1.5 We define the $\mathbb{Z}_{2}$-valued invariant

$$
\begin{aligned}
\sigma: \mathbb{G}(\Sigma) & \rightarrow \mathbb{Z}_{2} \\
\eta & \mapsto \begin{cases}0 & \text { if }\left[w_{\eta}\right]^{(2)}=0 \\
1 & \text { otherwise }\end{cases}
\end{aligned}
$$

We have a natural map induced by the inclusion $\partial \Sigma \rightarrow \Sigma$,

$$
i: H_{1}\left(\partial \Sigma ; \mathbb{Z}_{2}\right) \cong \mathbb{Z}_{2}^{d} \rightarrow H_{1}\left(\Sigma ; \mathbb{Z}_{2}\right) \cong \mathbb{Z}_{2}^{2 g+d-1}
$$

Note that the image of $i$ is precisely the kernel of the intersection pairing on $H_{1}\left(\Sigma, \mathbb{Z}_{2}\right)$, and the induced pairing on the cokernel of $i$ is non-degenerate. In fact, this cokernel is 
naturally isomorphic to $H_{1}\left(\bar{\Sigma} ; \mathbb{Z}_{2}\right) \simeq \mathbb{Z}_{2}^{2 g}$, where $\bar{\Sigma}$ is the surface without boundary obtained from $\Sigma$ by capping off all the boundary components.

Note that the values of $\left[w_{\eta}\right]^{(2)}$ on the boundary cycles are given by $w_{\eta}\left(\partial_{i} \Sigma\right)$ modulo 2. Thus, if at least one of these numbers is odd then $\sigma(\eta)=1$. If all the boundary winding numbers are even then we can check whether $\sigma(\eta)=0$ by looking at the winding numbers of a collection of cycles that form a basis in the homology of $\bar{\Sigma}$.

Proposition 1.6 Suppose $\eta$ is a line field on $\Sigma$ defined by the class $[\eta] \in H^{1}(\mathbb{P}(T \Sigma))$. There is a well defined map

$$
q_{\eta}: H_{1}\left(\Sigma ; \mathbb{Z}_{4}\right) \rightarrow \mathbb{Z}_{4}
$$

given by

$$
q_{\eta}\left(\sum_{i=1}^{m} \alpha_{i}\right)=\sum_{i=1}^{m} w_{\eta}\left(\alpha_{i}\right)+2 m \in \mathbb{Z}_{4},
$$

where $\alpha_{i}$ are simple closed curves. It satisfies

$$
q_{\eta}(a+b)=q_{\eta}(a)+q_{\eta}(b)+2(a \cdot b) \in \mathbb{Z}_{4}
$$

where $a, b \in H_{1}\left(\Sigma ; \mathbb{Z}_{4}\right)$, and $a \cdot b$ denotes the intersection pairing on $H_{1}\left(\Sigma ; \mathbb{Z}_{4}\right)$.

Proof In the case when $\eta$ comes from a non-vanishing vector field $v$, we have $w_{\eta}(a)=$ $2 w_{v}(a)$, where $w_{v}(\cdot)$ is the winding number of the vector field. Hence, the assertion in this case follows from [17, Thm 1A, Thm 1B]. In general, we have $\eta=\eta_{0}+c$, where $\eta_{0}$ comes from a non-vanishing vector field (which exists because $\Sigma$ is non-compact) and $c$ is a class in $H^{1}(\Sigma)$. Thus, the function $q_{\eta}(a):=q_{\eta_{0}}(a)+\langle c, a\rangle$ has the claimed properties.

Lemma 1.7 Suppose that $g(\Sigma) \geq 2$. Assume that line fields $\eta$ and $\theta$ have $w_{\eta}\left(\partial_{i} \Sigma\right)=$ $w_{\theta}\left(\partial_{i} \Sigma\right)$ for $i=1, \ldots, d$, and $q_{\eta}=q_{\theta}$. Then their homotopy classes lie in the same $\mathcal{M}(\Sigma)$-orbit.

Proof The assumption $q_{\eta}=q_{\theta}$ implies that $w_{\eta}(a) \equiv w_{\theta}(a) \bmod 4$ for any $a \in$ $H_{1}(\Sigma)$. Thus, we have $\theta=\eta+4 c$ for some $c \in H^{1}(\Sigma)$. Furthermore, the condition $w_{\eta}\left(\partial_{i} \Sigma\right)=w_{\theta}\left(\partial_{i} \Sigma\right)$ implies that $c$ has zero restriction to $H_{1}(\partial \Sigma)$. Hence, there exists $\alpha \in H_{1}(\Sigma)$, such that $\langle c, \gamma\rangle=(\alpha \cdot \gamma)$ for any $\gamma \in H_{1}(\Sigma)$. Now the fact that $\eta$ and $\theta$ lie in the same $\mathcal{M}(\Sigma)$-orbit is proved in exactly the same way as in the proof of [18, Thm. 2.5]. Namely, for each standard generator of the homology, $\alpha$, one can construct an explicit element in the mapping class $f_{\alpha}$ (expressed in terms of Dehn twists along certain curves related to $\alpha$ ) such that the action of $f_{\alpha}$ on a line field has the same effect as adding the class dual to $4 \alpha$.

Thus, for $g(\Sigma) \geq 2$, the study of the $\mathcal{M}(\Sigma)$-orbits on $G(\Sigma)$ reduces to the study of $\mathcal{M}(\Sigma)$-orbits on the set of functions $q: H_{1}\left(\Sigma, \mathbb{Z}_{4}\right) \rightarrow \mathbb{Z}_{4}$ satisfying

$$
q(a+b)=q(a)+q(b)+2(a \cdot b)
$$


Let us denote by Quad ${ }_{4}=\operatorname{Quad}_{4}(\Sigma)$ the set of all such functions (it is an $H^{1}\left(\Sigma, \mathbb{Z}_{4}\right)$ torsor).

Recall that given a symplectic vector space $V,(-\cdot-)$ over $\mathbb{Z}_{2}$, one can consider the set $\operatorname{Quad}(V)$ of quadratic forms $\bar{q}: V \rightarrow \mathbb{Z}_{2}$ satisfying

$$
\bar{q}(x+y)=\bar{q}(x)+\bar{q}(y)+(x \cdot y) .
$$

For every $\bar{q} \in \mathrm{Quad}(V)$, the Arf-invariant ([2,12]) is the element of $\mathbb{Z}_{2}$ given by

$$
A(\bar{q})=\sum_{i=1}^{n} \bar{q}\left(a_{i}\right) \bar{q}\left(b_{i}\right),
$$

where $\left(a_{i}, b_{i}\right)$ is a symplectic basis of $V$. The Arf invariant is the value that $\bar{q}$ attains on the majority of vectors in $V$.

In the case when $w_{\eta}\left(\partial_{i} \Sigma\right) \equiv 2 \bmod 4$ for every $i=1, \ldots, d$, and the quadratic function $q=q_{\eta}$ takes values in $2 \mathbb{Z}_{4}$, we can associate to $q$ an element in $\operatorname{Quad}\left(H_{1}\left(\bar{\Sigma}, \mathbb{Z}_{2}\right)\right)$ whose Arf-invariant will give us an additional invariant of $\eta$ modulo the mapping class group action.

Namely, it is easy to see that if $q \in$ Quad $_{4}$ takes values in $2 \mathbb{Z}_{4}$ then we have a well defined function $q / 2: H_{1}\left(\Sigma, \mathbb{Z}_{2}\right) \rightarrow \mathbb{Z}_{2}$ satisfying (1.5) such that $q=2 \cdot q / 2$. Now the condition $w_{\eta}\left(\partial_{i} \Sigma\right) \equiv 2 \bmod 4$ is equivalent to $q / 2\left(\partial_{i} \Sigma\right)=0$, so this is precisely the condition for the quadratic function $q / 2$ to descend to a form $\bar{q}$ in $\operatorname{Quad}\left(H_{1}\left(\bar{\Sigma}, \mathbb{Z}_{2}\right)\right.$ ) (recall that $H_{1}\left(\bar{\Sigma}, \mathbb{Z}_{2}\right)$ is the quotient of $H_{1}\left(\Sigma, \mathbb{Z}_{2}\right)$ by the boundary classes).

Thus, in the case when $\sigma(\eta)=0$ and $w_{\eta}\left(\partial_{i} \Sigma\right) \equiv 2 \bmod 4$ for every $i=1, \ldots, d$, we can apply the above construction to $q_{\eta}$ and define the quadratic form $\bar{q}_{\eta}$ in $\operatorname{Quad}\left(H_{1}\left(\bar{\Sigma}, \mathbb{Z}_{2}\right)\right)$. In this case we set

$$
A(\eta):=A\left(\bar{q}_{\eta}\right)
$$

In the case $g(\Sigma)=1$ we will use a different invariant of a line field, $\widetilde{A}(\eta)$, defined by

$$
\widetilde{A}(\eta):=\operatorname{gcd}\left(\left\{w_{\eta}(\alpha), w_{\eta}(\beta), w_{\eta}\left(\partial_{1} \Sigma\right)+2, \ldots, w_{\eta}\left(\partial_{d} \Sigma\right)+2\right\}\right)
$$

where $\alpha, \beta$ are non-separating curves in $\Sigma$ such that $[\alpha]$ and $[\beta]$ project to a basis of $H_{1}(\Sigma) / \mathrm{im}\left(i_{*}\right)$. It can be shown as in [18, Lemma 2.6] that

$$
\widetilde{A}(\eta)=\operatorname{gcd}\left(\left\{w_{\eta}(\gamma): \gamma \text { non-separating }\right\}\right)
$$

which implies that $\widetilde{A}(\cdot)$ is indeed invariant under the mapping class group. We also note that in the case $d=1, w_{\eta}(\partial \Sigma)=-2$, hence this invariant reduces to $\operatorname{gcd}\left(w_{\eta}(\alpha), w_{\eta}(\beta)\right)$ considered in [1]. 
Theorem 1.8 (i) Suppose $g(\Sigma)=0$. Then the action of $\mathcal{M}(\Sigma)$ on $G(\Sigma)$ is trivial. Moreover, two line fields $\eta$ and $\theta$ are homotopic if and only if

$$
w_{\eta}\left(\partial_{i} \Sigma\right)=w_{\theta}\left(\partial_{i} \Sigma\right) \text { for all } i=1, \ldots d .
$$

(ii) Suppose $g(\Sigma)=1$. Then two line fields $\eta$ and $\theta$ are in the same $\mathcal{M}(\Sigma)$-orbit if and only if

$$
w_{\eta}\left(\partial_{i} \Sigma\right)=w_{\theta}\left(\partial_{i} \Sigma\right) \text { for all } i=1, \ldots d \text {. }
$$

and

$$
\widetilde{A}(\eta)=\widetilde{A}(\theta) \in \mathbb{Z}_{\geq 0},
$$

where $\widetilde{A}(\eta)$ is defined by (1.6).

(iii) Suppose $g(\Sigma) \geq 2$. Then two line fields $\eta$ and $\theta$ are in the same $\mathcal{M}(\Sigma)$ orbit if and only if the following conditions are satisfied:

(1) $w_{\eta}\left(\partial_{i} \Sigma\right)=w_{\theta}\left(\partial_{i} \Sigma\right)$ for all $i=1, \ldots d$;

(2) $\sigma(\eta)=\sigma(\theta)$ (this only needs to be checked if all $w_{\eta}\left(\partial_{i} \Sigma\right)$ are even);

(3) if $w_{\eta}\left(\partial_{i} \Sigma\right)=w_{\theta}\left(\partial_{i} \Sigma\right) \in 2+4 \mathbb{Z}$ and $\sigma(\eta)=\sigma(\theta)=0$ then additionally one must have

$$
A(\eta)=A(\theta),
$$

where $A$ is an Arf invariant defined above.

Proof (i) This follows immediately from the fact that $G(\Sigma)$ is an $H^{1}(\Sigma)$-torsor and the boundary curves $\partial_{i} \Sigma$ generate the group $H_{1}(\Sigma)$.

(ii) This is proved in the same way as Theorem 2.8 in [18]. The main idea is to use the fact that for the standard choice of simple curves $\alpha$ and $\beta$, the Dehn twists with respect to $\alpha$ and $\beta$ generate an action of $\mathrm{SL}_{2}(\mathbb{Z})$ on the pair $\left(w_{\eta}(\alpha), w_{\eta}(\beta)\right)$ (one also uses some other Dehn twists, as in the proof of [18, Thm. 2.8]).

(iii) We need to prove that if the invariants match then $\eta$ and $\theta$ are in the same $\mathcal{M}(\Sigma)$ orbit. Note that $\sigma(\eta)$ is determined by whether the quadratic function $q_{\eta}$ is trivial modulo 2 or not. By Lemma 1.7, it is enough to prove that the quadratic functions $q_{\eta}$ and $q_{\theta}$ are in the same $\mathcal{M}(\Sigma)$-orbit.

First, let us analyze the result of the action of a transvection

$$
T_{a}(x)=x+(a \cdot x) a
$$

on quadratic functions in Quad 4 . Note that all such transvections can be realized by elements of the mapping class group: if the class $a$ is not divisible by 2 then we can lift it to a primitive element of the homology, and hence, $T_{a}$ is realized by some Dehn twist. On the other hand, if $a$ is divisible by 2 then $T_{a}=\mathrm{id}$. 
We have

$$
q\left(T_{a}(x)\right)=q(x)+(a \cdot x) q(a)+2(a \cdot x)(x \cdot a)=q(x)+(q(a)+2)(a \cdot x)(1.7)
$$

In particular, if $q(a)=-1$ then $q\left(T_{a}(x)\right)=q(x)+(a \cdot x)$.

Let us set

$$
H:=H_{1}\left(\Sigma, \mathbb{Z}_{4}\right), \quad K=\operatorname{im}\left(i_{*}: H_{1}\left(\partial \Sigma, \mathbb{Z}_{4}\right) \rightarrow H_{1}\left(\Sigma, \mathbb{Z}_{4}\right)\right) .
$$

If $q, q^{\prime} \in \operatorname{Quad}_{4}$ have $\left.q\right|_{K}=\left.q^{\prime}\right|_{K}$ then $\left(q^{\prime}-q\right)$ is a homomorphism $H \rightarrow \mathbb{Z}_{4}$, vanishing on $K$, hence it has form $x \mapsto(a \cdot x)$ for some $a \in H$.

Assume now that $q \in \operatorname{Quad}_{4}$ is such that $\left.q\right|_{K}$ is surjective, i.e., the reduction of $\left.q\right|_{K}$ modulo 2 is nonzero. Then we claim that any $q^{\prime} \in \operatorname{Quad}_{4}$ with $\left.q^{\prime}\right|_{K}=\left.q\right|_{K}$ lies in the $\mathcal{M}(\Sigma)$-orbit of $q$. Indeed, we have $q^{\prime}(x)-q(x)=(a \cdot x)$ for some $a \in H$. By surjectivity of $\left.q\right|_{K}$ we can find $k \in K$ such that $q(k)=-1-q(a)$, i.e., $q(a+k)=-1$. Then from (1.7) we get

$$
q T_{a+k}=q^{\prime}
$$

Next, let us consider $q \in \operatorname{Quad}_{4}$ such that $\left.q\right|_{K}$ takes values in $2 \mathbb{Z}_{4}$. Assume also that $q \bmod 2 \neq 0$. We claim that in this case the $\mathcal{M}(\Sigma)$-orbit of $q$ is determined by $\left.q\right|_{K}$. Note that $q \bmod 2$ is a homomorphism $H \rightarrow \mathbb{Z}_{2}$ trivial on $K$, so it is an element of $\operatorname{Hom}\left(H / K, \mathbb{Z}_{2}\right)$. Since $\mathcal{M}(\Sigma)$ acts transitively on nonzero elements in $\operatorname{Hom}\left(H / K, \mathbb{Z}_{2}\right)$, it is enough to prove that if $q^{\prime} \equiv q \bmod 2$ and $\left.q^{\prime}\right|_{K}=\left.q\right|_{K}$ then $q^{\prime}$ and $q$ are in the same $\mathcal{M}(\Sigma)$-orbit. As before we deduce that $q^{\prime}(x)-q(x)=2(a \cdot x)$ for some $a \in H$. If $q(a) \equiv 1 \bmod 2$ then this immediately gives $q^{\prime}=q T_{a}^{2}$. On the other hand, if $q^{\prime}(a) \equiv q(a) \equiv 0 \bmod 2$ then for any element $b$ with $q(b) \equiv 1 \bmod 2$ we have

$$
q T_{a+b}^{2}(x)=q(x)+2((a+b) \cdot x)=q^{\prime}(x)+2(b \cdot x)=q^{\prime} T_{b}^{2}(x),
$$

so $q^{\prime}$ and $q$ are in the same orbit.

Finally, if $q$ takes values in $2 \mathbb{Z}_{4}$ then we have $q=2 \cdot q / 2$ for a quadratic form $q / 2$ on $H / 2 H$ satisfying (1.5), and we can use the description of $\mathcal{M}(\Sigma)$-orbits on such forms from [18, Thm. 1.3] (based on the work of Johnson [17]).

Remark 1.9 1. It follows from (1.3) that the genus of the surface is determined by the boundary winding numbers of $\eta$ via the formula

$$
4-4 g(\Sigma)=\sum_{i=1}^{d}\left(w_{\eta}\left(\partial_{i} \Sigma\right)+2\right) .
$$

2. In the case $\sigma(\eta)=0$, the line field $\eta$ is induced by a non-vanishing vector field $v$ (see Lemma 1.4). This induces a spin structure on the surface $\Sigma$ (by considering its $\bmod 2$ reduction). The condition that $w_{\eta}\left(\partial_{i} \Sigma\right) \equiv 2 \bmod 4$, for $i=1, \ldots, d$, 
means that this spin structure extends to a spin structure on the compact surface obtained from $\Sigma$ by capping off the boundaries with a disk. Now, it is a theorem of Atiyah [4] (see also [17]) that the action of the mapping class group on the spin structures on a compact Riemann surface has exactly 2 orbits distinguished by the Arf invariant.

Theorem 1.8 can be used to get a criterion for a homeomorphism between two different graded surfaces.

Corollary 1.10 Let $\left(\Sigma_{1}, \Lambda_{1} ; \eta_{1}\right)$ and $\left(\Sigma_{2}, \Lambda_{2} ; \eta_{2}\right)$ be graded surfaces (where $\Lambda_{i}$ are sets of marked points on the boundary and $\eta_{i}$ are line fields) with the same number of boundary components $d$. Then there exists an orientation preserving homeomorphism $\phi: \Sigma_{1} \rightarrow \Sigma_{2}$ such that $\phi\left(\Lambda_{1}\right)=\Lambda_{2}$ and $\phi_{*}\left(\eta_{1}\right)$ is homotopic to $\eta_{2}$ if and only if there exists a numbering of boundary components on $\Sigma_{1}$ and $\Sigma_{2}$ such that for each $i=1, \ldots, d$, one has

$$
\begin{aligned}
\#\left(\Lambda_{1} \cap \partial_{i} \Sigma_{1}\right) & =\#\left(\Lambda_{2} \cap \partial_{i} \Sigma_{2}\right), \\
w_{\eta_{1}}\left(\partial_{i} \Sigma_{1}\right) & =w_{\eta_{2}}\left(\partial_{i} \Sigma_{2}\right),
\end{aligned}
$$

and in addition,

- if $g\left(\Sigma_{1}\right)=g\left(\Sigma_{2}\right)=1$ then $\widetilde{A}\left(\eta_{1}\right)=\widetilde{A}\left(\eta_{2}\right)$;

- if $g\left(\Sigma_{1}\right)=g\left(\Sigma_{2}\right) \geq 2$ then $\sigma\left(\eta_{1}\right)=\sigma\left(\eta_{2}\right)$ and $A\left(\eta_{1}\right)=A\left(\eta_{2}\right)$ whenever the latter two invariants are defined.

Proof The "only if" part is clear. For the "if" part, since $g\left(\Sigma_{1}\right)=g\left(\Sigma_{2}\right)$ [due to (1.8)], we can find a homeomorphism $\widetilde{\phi}: \Sigma_{1} \rightarrow \Sigma_{2}$ sending $\partial_{i} \Sigma_{1}$ to $\partial_{i} \Sigma_{2}$ and $\Lambda_{1}$ to $\Lambda_{2}$. Applying Theorem 1.8 to $\widetilde{\phi}_{*} \eta_{1}$ and $\eta_{2}$ we deduce the existence of an element $\psi \in$ $\mathcal{M}\left(\Sigma_{2}\right)$ such that $\psi_{*}\left(\widetilde{\phi}_{*} \eta_{1}\right)=\eta_{2}$. Thus, the homeomorphism $\phi=\psi \circ \widetilde{\phi}: \Sigma_{1} \rightarrow \Sigma_{2}$ has the required properties.

\section{Partially wrapped Fukaya categories}

The partially wrapped Fukaya category $\mathcal{W}(\Sigma, \Lambda ; \eta)$ (with coefficients in a field $\mathbb{K}$ ) is associated to a graded surface $(\Sigma, \Lambda ; \eta)$, where $\Sigma$ is a connected compact surface with non-empty boundary $\partial \Sigma, \Lambda \subset \partial \Sigma$ is a collection of marked points called stops, and $\eta$ is a line field on $\Sigma$. Partially wrapped Fukaya categories were first introduced in the work of Auroux [5] in arbitrary dimension. In the case the symplectic manifold is a surface, which is our focus in this paper, there is a combinatorial description of $\mathcal{W}(\Sigma, \Lambda ; \eta)$ provided in [15]. The latter not only gives a topological computation of the partially wrapped Fukaya category defined by symplectic machinery in [5], but also provides an independent, purely topological proof of the invariance of $\mathcal{W}(\Sigma, \Lambda ; \eta)$ using the well-known contractibility result of Harer's arc complex. In particular, it follows from this topological description that given two graded surfaces with stops, $\left(\Sigma_{i}, \Lambda_{i}, \eta_{i}\right)$ for $i=1,2$, a homeomorphism $\phi: \Sigma_{1} \rightarrow \Sigma_{2}$ which restricts to a bijection $\Lambda_{1} \rightarrow \Lambda_{2}$ and a homotopy between $\phi_{*}\left(\eta_{1}\right)$ to $\eta_{2}$, we get an equivalence 
between the partially wrapped Fukaya categories $\mathcal{W}\left(\Sigma_{1}, \Lambda_{1} ; \eta_{1}\right)$ and $\mathcal{W}\left(\Sigma_{2}, \Lambda_{2} ; \eta_{2}\right)$. The proof of the equivalence of the two approaches, [5] and [15] given by Abouzaid in the case $\Lambda=\emptyset$ in the appendix of [10] easily extends to the general case. Another possible approach to this equivalence is via the definition of wrapped Fukaya categories given in [13] which uses the symplectic field theory formulation. We note that we do not need to appeal to any of these equivalences for the applications in this paper, we simply work with with the definition and the established results given in [15]. We next recall this combinatorial description of the partially wrapped Fukaya categories from [15].

A set of pairwise disjoint and non-isotopic Lagrangians $\left\{L_{i}\right\}$ in $\Sigma \backslash \Lambda$ generates the partially wrapped Fukaya category $\mathcal{W}(\Sigma, \Lambda ; \eta)$ as a triangulated category if the complement of the Lagrangians

$$
\Sigma \backslash\left\{\bigsqcup_{i} L_{i}\right\}=\bigcup_{f} D_{f}
$$

is a union of disks $D_{f}$ each of which has at most one stop on its boundary. Furthermore, if each $D_{f}$ has exactly one stop in its boundary, the associative $\mathbb{K}$-algebra

$$
A_{L_{\bullet}}:=\bigoplus_{i, j} \operatorname{hom}\left(L_{i}, L_{j}\right)
$$

is formal, and it can be described by a graded gentle algebra (see Definition 3.1). Figure 1 illustrates how each $D_{f}$ may look like, where the blue arcs are in $\bigsqcup_{i} L_{i}$ while the black arcs lie in $\partial \Sigma$.

The algebra $A_{L}$. can easily be described by a quiver following the flow lines corresponding to rotation around the boundary components of $\Sigma$ connecting the Lagrangians. Note that each boundary component of $\Sigma$ is an oriented circle (where the boundary orientation is induced by the area form on $\Sigma$ ). Specifically, a flowline that goes from $L_{j}$ to $L_{i}$ gives a generator for hom $\left(L_{i}, L_{j}\right)$ (note the reversal of indices). The data of $\Lambda$ enters by disallowing flows that pass through a marked point. The algebra structure is given by concatenation of flow lines. Given $\alpha_{i} \in \operatorname{hom}\left(L_{i}, L_{i+1}\right)$ for $i=1, \ldots, n$, we write

Fig. 1 An example of a disk $D_{f}$

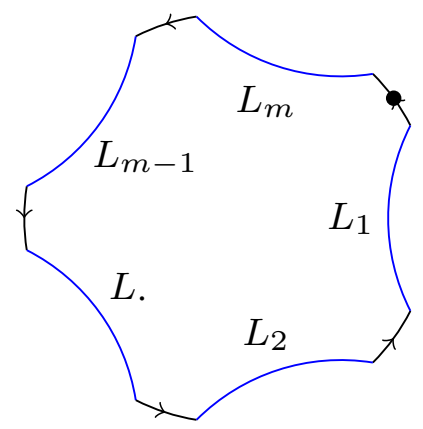




$$
\alpha_{n} \alpha_{n-1} \ldots \alpha_{1} \in \operatorname{hom}\left(L_{1}, L_{n+1}\right)
$$

for their product, read from right to left, and if non-zero, this expression corresponds to a flow from $L_{n+1}$ to $L_{1}$.

Finally, the line field $\eta$ is used to grade the morphism spaces. A convenient way to determine the line field $\eta$ is by describing its restrictions along each of the disks $D_{f}$. Each such disk is as in Fig. 1. Different disks are glued along the curves $L_{i}$ (the blue parts in their boundary). As $L_{i}$ are contractible, changing a line field by homotopy, we can arrange that it is transverse to $L_{i}$ everywhere along $L_{i}$. Every line field on $\Sigma$ (up to homotopy) can be glued out of such line fields on the disks $D_{f}$.

Note that if we have an embedded segment $c \subset \Sigma$ and a line field $\eta$, which is transversal to $c$ at the ends $p_{1}, p_{2}$ of $c$, then we can define the winding number $w_{\eta}(c)$ (first, one can trivialize $T \Sigma$ along $c$ in such a way that the tangent line to $c$ is constant, then count the number of times (with sign) $\eta$ coincides with the tangent line to $c$ along $c$. An equivalent definition is given in [15, Sec. 3.2]). Now a line field on a disk $D_{f}$, transverse to $\left\{L_{i}\right\}$, is determined (up to homotopy) by the integers $\theta_{i}$, for $i=1, \ldots, m$, given by its winding numbers along the boundary parts on $\partial \Sigma$ (the black parts in Fig. 1). By definition, these numbers are the degrees of the corresponding morphisms in the wrapped Fukaya category.

The numbers $\theta_{i}$ can be chosen arbitrarily subject to the constraint

$$
\sum_{i=1}^{m} \theta_{i}=m-2 .
$$

This last constraint is the topological condition that needs to be satisfied in order for the line field to extend to the interior of the disk. (Note that the stops do not play a role in this discussion.)

The gentle algebra $A_{L_{\bullet}}$ is always homologically smooth since so is $\mathcal{W}(\Sigma, \Lambda ; \eta)$. The algebra $A_{L}$ is proper (i.e., finite-dimensional) if and only if there is at least one marked point on every boundary component. The "if" part is [15, Cor. 3.1]. On the other hand, if there is a boundary component with no stops, then we can compose flows along this boundary indefinitely, so $A_{L_{\bullet}}$ is not proper.

In what follows, it will be convenient to consider $A_{L_{\bullet}}^{o p}$ as a quiver algebra $\mathbb{K} Q / I$, so that flow lines from $L_{i}$ to $L_{j}$ correspond to arrows from the $i^{\text {th }}$ vertex to $j^{\text {th }}$ vertex. Note that the collection $\left\{L_{i}\right\}$ generates the partially wrapped Fukaya category $\mathcal{W}(\Sigma, \Lambda ; \eta)$. Therefore, we have an equivalence

$$
D\left(A_{L_{\bullet}}^{o p}\right) \cong \mathcal{W}(\Sigma, \Lambda ; \eta)
$$

where the category on the left denotes the bounded derived category of perfect (left) dg-modules over $A_{L_{\bullet}}^{o p}$. 


\section{Gentle algebras and Fukaya categories}

\subsection{Graded gentle algebras and AAG-invariants}

A quiver is a tuple $Q=\left(Q_{0}, Q_{1}, s, t\right)$ where $Q_{0}$ is the set of vertices, $Q_{1}$ is the set of arrows, $s, t: Q_{1} \rightarrow Q_{0}$ is the functions that determine the source and target of the arrows. We always assume $Q$ to be finite. A path in $Q$ is a sequence of arrows $\alpha_{n} \ldots \alpha_{2} \alpha_{1}$ such that $s\left(\alpha_{i+1}\right)=t\left(\alpha_{i}\right)$ for $i=1, \ldots,(n-1)$. A cycle in $Q$ is a path of length $\geq 1$ in which the beginning and the end vertices coincide but otherwise the vertices are distinct. For $\mathbb{K}$ a field, let $\mathbb{K} Q$ be the path algebra, with paths in $Q$ as a basis and multiplication induced by concatenation. Note that the source $s$ and target $t$ maps have obvious extensions to paths in $Q$.

Definition 3.1 A gentle algebra ${ }^{2} A=\mathbb{K} Q / I$ is given by a quiver $Q$ with relations $I$ such that

(1) Each vertex has at most two incoming and at most two outgoing edges.

(2) The ideal $I$ is generated by composable paths of length 2 .

(3) For each arrow $\alpha$, there is at most one arrow $\beta$ such that $\alpha \beta \in I$ and there is at most one arrow $\beta$ such that $\beta \alpha \in I$.

(4) For each arrow $\alpha$, there is at most one arrow $\beta$ such that $\alpha \beta \notin I$ and there is at most one arrow $\beta$ such that $\beta \alpha \notin I$.

In addition, we always assume $Q$ to be connected.

We will consider $\mathbb{Z}$-graded gentle algebras, i.e., every arrow in $Q$ should have a degree assigned to it. For a $\mathbb{Z}$-graded gentle algebra $A$ we denote by $D(A)$ the derived category of perfect $\mathrm{dg}$-modules over $A$, where $A$ is viewed as a dg-algebra with its natural grading and zero differential.

Remark 3.2 Note that $D(A)$ is different from the derived category of graded $A$ modules. In fact, the former category is obtained from the latter as a suitable orbit category (see the discussion in [24, Sec. 1.3]). On the other hand, it is well known that if the grading of $A$ is zero then $D(A)$ is equivalent to the perfect derived category of ungraded $A$-modules. Indeed, in this case a $\operatorname{dg}$-module over $A$ is the same thing as a complex of $A$-modules.

Lemma 3.3 (i) A gentle algebra is homologically smooth if and only if there are no forbidden cycles i.e. cycles $\alpha_{n} \ldots \alpha_{2} \alpha_{1}$ in $\mathbb{K} Q$ such that $\alpha_{i+1} \alpha_{i} \in I$ for $i \in \mathbb{Z} / n$.

(ii) A gentle algebra is proper (i.e., finite-dimensional) if and only if there are no permitted cycles i.e. paths $\alpha_{n} \ldots \alpha_{2} \alpha_{1}$ in $\mathbb{K} Q$ such that $\alpha_{i+1} \alpha_{i} \notin I$ for $i \in \mathbb{Z} / n$.

Proof The "if" direction is proved in [15, Prop. 3.4(1)] using an explicit form of the resolution of the diagonal bimodule. Note that such a resolution goes back to Bardzell's work [8] (where the case of arbitrary monomial relations is considered). It

2 Our terminology is the same as in [25], so we do not impose the condition of finite-dimensionality in the definition of a gentle algebras. What we call "gentle algebra" is sometimes referred to as "locally gentle algebra". 
remains to prove that if a gentle algebra $A$ is homologically smooth then there are no forbidden cycles. Since $A$ is homologically smooth, the diagonal bimodule is a perfect dg-module over $A^{o p} \otimes A$. Thus, for every simple $A$-module $S$ (corresponding to one of the vertices), we get a quasi-isomorphism of $S$ with a perfect dg-module over $A$. It follows that the space $\operatorname{Ext}_{A-\operatorname{dgmod}}^{*}(S, S)$ is finite-dimensional. Equivalently, the space $\operatorname{Ext}_{A}^{*}(S, S)$, computed in the category of ungraded $A$-modules, is finite-dimensional (see [24, Thm. 1.3.3]). But the latter space can be computed using the standard Koszul complex, and the presence of forbidden cycles would mean that for some $S$ the space $\operatorname{Ext}_{A}^{*}(S, S)$ is infinite-dimensional.

(ii) This is straightforward as properness is equivalent to having only finite number of paths that are nonzero in $A$ (see [15, Prop. 3.4(2)]).

We will use the following notions from [7].

Definition 3.4 A forbidden path is a path in $Q$ of the form

$$
f=\alpha_{n-1} \ldots \alpha_{2} \alpha_{1} \in \mathbb{K} Q
$$

such that all $\left(\alpha_{i}\right)$ are distinct and for all $i=1, \ldots,(n-2), \alpha_{i+1} \alpha_{i} \in I$. It is a forbidden thread if for all $\beta \in Q_{1}$ neither $\beta \alpha_{n} \ldots \alpha_{2} \alpha_{1}$ nor $\alpha_{n} \ldots \alpha_{2} \alpha_{1} \beta$ is a forbidden path. In addition, if $v \in Q_{0}$ with $\#\left\{\alpha \in Q_{1} \mid s(\alpha)=v\right\} \leq 1, \#\left\{\alpha \in Q_{1} \mid t(\alpha)=v\right\} \leq 1$, then we consider the idempotent $e_{v}$ as a (trivial) forbidden thread in the following cases:

- either there are no $\alpha$ with $s(\alpha)=v$ or there are no $\alpha$ with $t(\alpha)=v$;

- we have $\beta, \gamma \in Q_{1}$ with $s(\gamma)=v=t(\beta)$ and $\gamma \beta \in I$.

The grading of a forbidden thread is defined by

$$
|f|=\sum_{i=1}^{n-1}\left|\alpha_{i}\right|-(n-2) .
$$

Definition 3.5 A permitted path is a path in $Q$ of the form

$$
p=\alpha_{n} \ldots \alpha_{2} \alpha_{1} \in \mathbb{K} Q
$$

such that all $\left(\alpha_{i}\right)$ are distinct and for all $i=1, \ldots,(n-1), \alpha_{i+1} \alpha_{i} \notin I$, and it is a permitted thread if for all $\beta \in Q_{1}$ neither $\beta \alpha_{n} \ldots \alpha_{2} \alpha_{1}$ nor $\alpha_{n} \ldots \alpha_{2} \alpha_{1} \beta$ is a permitted path. In addition, if $v \in Q_{0}$ with $\#\left\{\alpha \in Q_{1} \mid s(\alpha)=v\right\} \leq 1, \#\left\{\alpha \in Q_{1} \mid t(\alpha)=v\right\} \leq 1$, then we consider the idempotent $e_{v}$ as a (trivial) permitted thread in the following cases:

- either there are no $\alpha$ with $s(\alpha)=v$ or there are no $\alpha$ with $t(\alpha)=v$;

- we have $\beta, \gamma \in Q_{1}$ with $s(\gamma)=v=t(\beta)$ and $\gamma \beta \notin I$.

The grading of a permitted thread is defined by

$$
|p|=-\sum_{i=1}^{n}\left|\alpha_{i}\right|
$$


Remark 3.6 Inclusion of the idempotents as forbidden and permitted threads ensures that every vertex appears in exactly two forbidden threads/cycles and exactly two permitted threads/cycles.

Definition 3.7 For a gentle algebra A, a combinatorial boundary component of type I is an alternating cyclic sequence of forbidden and permitted threads:

$$
b=p_{n} f_{n} \ldots p_{2} f_{2} p_{1} f_{1}
$$

such that $s\left(f_{i}\right)=s\left(p_{i}\right)$ for $i \in \mathbb{Z} / n$, and $t\left(p_{i}\right)=t\left(f_{i+1}\right)$ for $i \in \mathbb{Z} / n$ with the following condition:

$(\star)$ For each $i \in \mathbb{Z} / n$, if $f_{i+1}=\alpha_{k} \ldots \alpha_{1}, p_{i}=\beta_{m} \ldots \beta_{1}$, and $f_{i}=\gamma_{n} \ldots \gamma_{1}$ such that $s\left(f_{i}\right)=s\left(p_{i}\right)$ and $t\left(p_{i}\right)=t\left(f_{i+1}\right)$, we have

$$
\gamma_{1} \neq \beta_{1} \quad \text { and } \quad \beta_{m} \neq \alpha_{k}
$$

The winding number associated to a combinatorial boundary component $b$ of type I is defined to be

$$
w(b):=\sum_{i=1}^{r}\left(\left|p_{i}\right|+\left|f_{i}\right|\right) .
$$

We also denote the number $n$ of forbidden threads in $b$ as $n(b)$.

A combinatorial boundary component of type II (that can appear only if $A$ is not proper) is simply a permitted cycle

$$
p c=\alpha_{m} \ldots \alpha_{1}
$$

The winding number associated to such a cycle is

$$
w(p c):=-\sum_{i=1}^{m}\left|\alpha_{i}\right| .
$$

A combinatorial boundary component of type II' (that can appear only if $A$ is not homologically smooth) is simply a forbidden cycle

$$
f c=\alpha_{m} \ldots \alpha_{1}
$$

The winding number associated to such a cycle is

$$
w(f c):=\sum_{i=1}^{m}\left|\alpha_{i}\right|-m .
$$

For combinatorial boundary components of types II and II' we set $n(b)=0$. 
Lemma 3.8 Let $A$ be a proper gentle algebra, with grading in degree zero. Then the collection of pairs $(n(b), n(b)-w(b))$, over all combinatorial boundary components (taken with multiplicities) coincides with AAG-invariants of $A$.

Proof This follows directly from the description of the AAG-invariants in [7, Sec. 3]. Note that the pair $(0, m)$ in Step (3) of the algorithm of [7, Sec. 3] associated to a forbidden cycle $f c=\alpha_{m} \ldots \alpha_{1}$ matches with the pair $(0, w(f c))$ associated with the corresponding combinatorial component of type II'. Indeed, $w(f c)=m$ since the grading of $A$ is in degree 0 .

From now on we will always assume that our gentle algebras are homologically smooth, with the exception of Remark 3.20.

Motivated by Lemma 3.8 we extend the definition of the AAG-invariants to graded gentle algebras.

Definition 3.9 For a graded gentle algebra $A$ we define the $A A G$-invariants to be the collection of pairs $(n(b), n(b)-w(b))$, taken with multiplicities, where $b$ runs over all combinatorial boundary components of $A$.

\subsection{Relation to Fukaya categories}

The definition of the combinatorial boundary component for a gentle algebra is motivated by the following proposition:

Proposition 3.10 Suppose $\Sigma$ is a surface with a collection of marked points $\Lambda \subset \partial \Sigma$, and a line field $\eta$. Let $\left\{L_{i}\right\}$ be a collection of Lagrangians such that the complement of $\bigsqcup_{i} L_{i}$ is a union of disks each of which has exactly one stop on its boundary. Then the combinatorial boundary components of the homologically smooth gentle algebra $A=\left(\bigoplus_{i, j} \operatorname{hom}\left(L_{i}, L_{j}\right)\right)^{o p}$ are in natural bijection with the boundary components of $\partial \Sigma$. Furthermore, if a combinatorial boundary component $b$ corresponds to a boundary component $B \subset \partial \Sigma$ then the number of forbidden threads in $b$ is equal to the number of stops on $B$ and the winding numbers match:

$$
w_{\eta}(B)=w(b)
$$

Proof Figure 2 shows an example of the way the surface $\Sigma$ looks around a boundary component $B$. Assume first that there is at least one stop on $B$. Let

$$
q_{1}(1), \ldots, q_{1}\left(k_{1}\right), q_{2}(1), \ldots, q_{2}\left(k_{2}\right), \ldots, q_{n}(1), \ldots, q_{n}\left(k_{n}\right)
$$

be the endpoints of the Lagrangians ending on $B$, ordered compatibly with the orientation of $B$. Here we assume that there are no stops between $q_{i}(j)$ and $q_{i}(j+1)$ and there is exactly one stop $s_{i}$ between $q_{i}\left(k_{i}\right)$ and $q_{i+1}(1)$, for $i \in \mathbb{Z} / n$. Then for every $i \in \mathbb{Z} / n$ we have a permitted thread $p_{i}=\beta_{i}\left(k_{i}-1\right) \ldots \beta_{i}(1)$, where $\beta_{i}(j)$ is the generator of $A$ corresponding to the flow on $B$ from $q_{i}(j)$ to $q_{i}(j+1)$. On the other hand, each stop $s_{i}$ lies on a unique disk $D$, and by looking at the pieces of $\partial D$ formed by other boundary components of $\Sigma$, we obtain a forbidden thread 
Fig. 2 The boundary component is given by the cyclic sequence $p_{2} f_{2} p_{1} f_{1}$ where $f_{1}=\alpha_{3} \alpha_{2} \alpha_{1}$, $p_{1}=\beta_{2} \beta_{1}, f_{2}=\gamma_{2} \gamma_{1}$ and $p_{2}=\delta_{1}$. Note that if instead of $f_{1}$, we considered the forbidden thread $\tilde{f}_{1}=\tilde{\beta}_{2} \beta_{1}$, the condition $\star \star)$ is violated

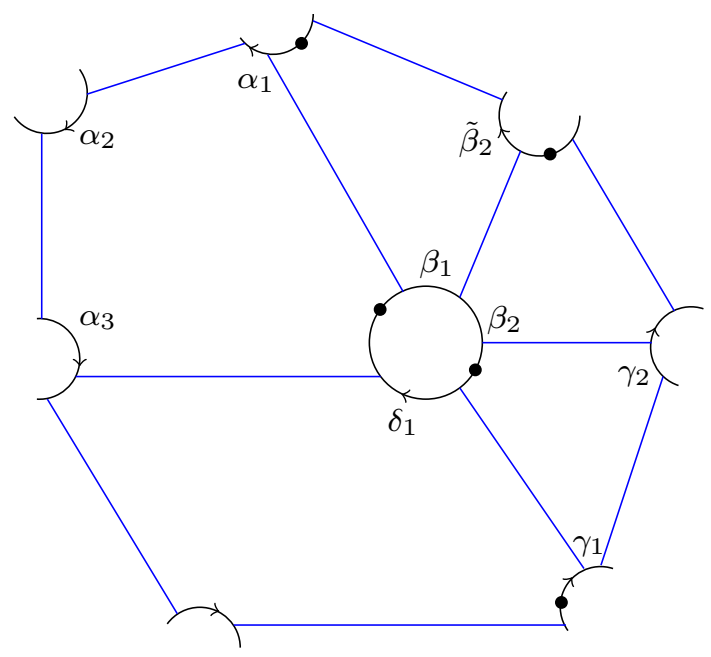

$f_{i}=\alpha_{m_{i}} \ldots \alpha_{1}$ starting at the Lagrangian corresponding to $q_{i}(1)$ and ending at the one corresponding to $q_{i-1}\left(k_{i-1}\right)$. Thus, we get a combinatorial boundary component of type I, $b=p_{n} f_{n} \ldots p_{1} f_{1}$.

The winding number of $\eta$ along the arc passing through the stop, oriented in the opposite direction to the boundary direction, is determined using the constraint (2.1) to be

$$
|f|=\sum_{i=1}^{n-1}\left|\alpha_{i}\right|-(n-2)
$$

On the other hand, the winding number of $\eta$ along the arc corresponding to the permitted thread $p$ is simply $|p|$. Thus, we get the equality $w_{\eta}(B)=w(b)$.

In the case of a boundary component $B \subset \partial \Sigma$ with no stops, the sequence of flows between the corresponding ends of Lagrangians on $B$ gives a permitted cycle, i.e., a combinatorial boundary component of type II. Again, the winding numbers match.

It is easy to see that in this way we get a bijection between the boundary components $B$ and the combinatorial boundary components of $A$.

Let $A$ be a homologically smooth gentle algebra. We associate with $A$ a ribbon graph $\mathcal{R}_{A}$ whose vertices are in bijection with the collection of forbidden threads in $Q$, and whose edges are in bijection with vertices of $Q$. More precisely, recall that there are precisely two forbidden threads that pass through a vertex of $Q$. The corresponding edge on $\mathcal{R}_{A}$ is defined to connect the two forbidden threads.

Next, we will define a ribbon structure, i.e., a cyclic order on the set of edges incident to each vertex. In fact, we will equip each such set of edges with a total order which will induce a cyclic order. (Thus, we get what is called a ciliated fat graph [14].) Namely, the set of edges incident to a vertex $f$ of $\mathcal{R}$ is in bijection with the set of vertices of $Q$ which appear in the forbidden thread $f$. Now we use the order in which these vertices appear in the forbidden thread $f$. 
Thus, we can consider the associated thickened surface $\Sigma_{A}$ such that $\mathcal{R}_{A}$ is embedded as a deformation retract of $\Sigma_{A}$. More specifically, to construct $\Sigma_{A}$ we replace each vertex of $\mathcal{R}_{A}$ with a 2 -disk $\mathbb{D}^{2}$ and each edge with a strip, a thin oriented rectangle $[-\epsilon, \epsilon] \times[0,1]$, where the rectangles are attached to the boundary of the disks according to the given cyclic orders at the vertices. On the boundary of each disk associated to the vertex of $\mathcal{R}_{A}$ we also mark a point, called a stop as follows. If the linear order on edges incident to this vertex is given by $e_{1}<e_{2}<\ldots<e_{k}$, the stop $e_{0}$ appears in the circular order such that $e_{k}<e_{0}<e_{1}$. We define $\Lambda_{A}$ by taking the union of all such points. In particular, the cardinality of $\Lambda_{A}$, is equal to the number of forbidden threads in $A$.

Theorem 3.11 (i) Given a homologically smooth gentle algebra A over a field $\mathbb{K}$ (with $\left|Q_{1}\right|>0$ ), let $\left(\Sigma_{A}, \Lambda_{A}\right)$ be the corresponding surface with stops defined above. Then $\Sigma_{A}$ is connected with non-empty boundary, and for each $\mathbb{Z}$-grading on A there is a natural line field $\eta$ on $\Sigma$ such that we have a derived equivalence

$$
D(A) \cong \mathcal{W}\left(\Sigma_{A}, \Lambda_{A} ; \eta_{A}\right)
$$

Furthermore, the AAG-invariants of A are given by the collection of pairs

$$
\left(n_{i}, n_{i}-w_{\eta_{A}}\left(\partial_{i} \Sigma_{A}\right)\right)
$$

where $\left.\left(\partial_{i} \Sigma_{A}\right)_{i=1, \ldots, N}\right)$ are all boundary components of $\Sigma_{A}$ and $n_{i} \in \mathbb{Z}_{\geq 0}$ is the number of marked points on $\partial_{i} \Sigma_{A}$.

(ii) One has

$$
\chi\left(\Sigma_{A}\right)=\chi(Q)=\left|Q_{0}\right|-\left|Q_{1}\right|
$$

Proof (i) First, let us check that the ribbon graph $\mathcal{R}_{A}$ and hence the associated surface $\Sigma_{A}$ is connected. Indeed, for every vertex $v$ of $Q$ let $e(v)$ be the corresponding edge in $\mathcal{R}_{A}$, viewed as a subgraph in $\mathcal{R}_{A}$. Since $Q$ is connected, it is enough to check that if $v$ and $v^{\prime}$ are connected by an edge $\alpha$ in $Q$ then $e(v)$ and $e\left(v^{\prime}\right)$ intersect in $\mathcal{R}_{A}$. Indeed, let $f$ be a forbidden thread containing $\alpha$ (it always exists). Then $f$ is a vertex of both $e(v)$ and $e\left(v^{\prime}\right)$. This proves our claim that $\mathcal{R}_{A}$ is connected.

Dual to the edges of $\mathcal{R}_{A}$ we obtain a disjoint collection of non-compact arcs $L_{v}$ indexed by vertices of $Q$. Thus, $\Sigma_{A}$ is a surface with non-empty oriented boundary, $\Lambda_{A}$ is a set of marked points in its boundary, and $\left\{L_{v}: v \in Q_{0}\right\}$ is a collection pair-wise disjoint and non-isotopic Lagrangian arcs in $\Sigma_{A} \backslash \Lambda_{A}$. Furthermore, the complement

$$
\Sigma_{A} \backslash\left\{\bigsqcup_{v} L_{v}\right\}=\bigcup_{f} D_{f}
$$

is a union of disks $D_{f}$ indexed by forbidden threads $f$ in $Q$, with exactly one marked point on its boundary (see Examples $3.17,3.18$ below). In particular, the collection $\left\{L_{v}\right\}$ gives a generating set. 
By construction, there is a bijection between arrows in the quiver $Q$ and the generators of the endomorphism algebra $A_{L}:=\bigoplus_{v, w} \operatorname{hom}\left(L_{v}, L_{w}\right)$ since each edge $\alpha$ in $Q$ is in exactly one forbidden thread $f$, and the corresponding $D_{f}$ has a flow associated to $\alpha$. Furthermore, two flows $\alpha_{1}: L_{v_{2}} \rightarrow L_{v_{1}}$ and $\alpha_{2}: L_{v_{3}} \rightarrow L_{v_{2}}$ can be composed in $A_{L}$ if and only if $\alpha_{i}$ is in a forbidden thread $f_{i}$, for $i=1,2$, such that the disks $D_{f_{1}}$ and $D_{f_{2}}$ are glued along the edge corresponding to $v_{2}$. But this means that the corresponding elements of $A$ satisfy $\alpha_{2} \alpha_{1} \notin I$, as otherwise condition (3) of Definition 3.1 would be violated. This implies that $A$ is naturally identified with $A_{L}^{o p}$ as an ungraded algebra.

We define the line field $\eta_{A}$ on $\Sigma_{A}$ as follows. We require that the line field is transverse to each $L_{v}$. Then it suffices to describe its restrictions to the disks $D_{f}$. Each $D_{f}$ is a $2 m$-gon as in Fig. 1. As explained in Sect. 2, the homotopy class of a line field on $D_{f}$ is determined by the winding numbers $\theta_{i}$ along the boundary arcs of $D_{f}, \alpha_{i}$, for $i=1, \ldots,(m-1)$, avoiding the unique stop (black in Fig. 1) between the Lagrangians (blue in Fig. 1). Indeed, the remaining winding number $\theta_{m}$ along the boundary arc that passes through the stop is determined by the condition $\sum_{i=1}^{m} \theta_{i}=m-2$, and we can define $\left.\eta_{A}\right|_{D_{f}}$ as the unique line field with these winding numbers. Now we set $\theta_{i}$, for $i=1, \ldots, m-1$, to be the degree of the generator of $A$ corresponding to $\alpha_{i}$.

With this definition $A$ and $A_{L}^{o p}$ are identified as graded algebras. Since we also know that the collection $\left\{L_{v}\right\}$ generates $\mathcal{W}\left(\Sigma_{A}, \Lambda_{A} ; \eta_{A}\right)$, we conclude that

$$
D(A) \cong \mathcal{W}\left(\Sigma_{A}, \Lambda_{A} ; \eta_{A}\right)
$$

Finally, the last statement follows from Proposition 3.10.

(ii) We have $\chi\left(\Sigma_{A}\right)=\chi\left(\mathcal{R}_{A}\right)$. Let us denote by $v\left(\mathcal{R}_{A}\right)$ and $e\left(\mathcal{R}_{A}\right)$ the numbers of vertices and edges in $\mathcal{R}_{A}$. We have $e\left(\mathcal{R}_{1}\right)=\left|Q_{0}\right|$, while $v\left(\mathcal{R}_{A}\right)$ is the number of forbidden threads. Let $f_{1}, \ldots, f_{m}$ be all forbidden threads. Since every edge of $Q$ belongs to the unique forbidden thread, we have

$$
\sum \ell\left(f_{i}\right)=\left|Q_{1}\right|
$$

(where $\ell(\cdot)$ is the length). On the other hand, since every vertex is contained in exactly two forbidden threads, we have

$$
\sum\left(\ell\left(f_{i}\right)+1\right)=2\left|Q_{0}\right|
$$

Combining this with the previous formula we get

$$
v\left(\mathcal{R}_{A}\right)=2\left|Q_{0}\right|-\left|Q_{1}\right|
$$

so we deduce that $\chi\left(\mathcal{R}_{A}\right)=\chi(Q)$.

Using formula (1.8) we derive the following property of the AAG-invariants. 
Corollary 3.12 Let $\left\{\left(n_{i}, m_{i}\right)\right\}_{i=1, \ldots, d}$ be the AAG-invariants of a homologically smooth graded gentle algebra $A$. Then

$$
\sum_{i=1}^{d}\left(n_{i}-m_{i}+2\right)=4-4 g
$$

where $g \geq 0$ is the genus of the corresponding surface $\Sigma_{A}$.

Combining Theorem 3.11 with Corollary 1.10, we get the following result.

Corollary 3.13 Given two homologically smooth graded gentle algebras $A$ and $B$, assume that the $A A G$-invariants of $A$ and $B$ are the same, and in addition, the invariants $\widetilde{A}(\cdot), \sigma(\cdot)$ and $A(\cdot)$ (see Theorem 1.8) of the line fields $\eta_{A}\left(\right.$ on $\left.\Sigma_{A}\right)$ and $\eta_{B}\left(\right.$ on $\left.\Sigma_{B}\right)$ are the same whenever they are defined. Then $D(A) \simeq D(B)$.

As a particular case of the last Corollary, we can describe some cases when already looking at the AAG-invariants gives the derived equivalence.

Corollary 3.14 Assume that $A$ and $B$ are homologically smooth graded gentle algebras, such that the $A A G$-invariants of $A$ and $B$ coincide (up to permutation) and are given by a collection $\left\{\left(n_{i}, m_{i}\right)\right\}_{i=1, \ldots, d}$. Assume in addition that one of the following conditions holds:

(a) $\sum_{i}\left(n_{i}-m_{i}+2\right)=4$;

(b) $\sum_{i}\left(n_{i}-m_{i}+2\right)=0$ and $\operatorname{gcd}\left(n_{1}-m_{1}+2, \ldots, n_{d}-m_{d}+2\right)=1$;

(c) $\sum_{i}\left(n_{i}-m_{i}+2\right)<0$ and at least one of the numbers $n_{i}-m_{i}$ is odd.

Then $D(A) \simeq D(B)$.

Proof By Corollary 3.12, the three cases are distinguished by the genus $g\left(\Sigma_{A}\right)$ : in case (a) it is 0 , in case (b) it is 1 , and in case (c) it is $>1$. Now the assertion follows from Corollary 1.10 .

Remark 3.15 There is a simple combinatorial recipe for calculating winding numbers of the line field $\eta$ on $\Sigma_{A}$, along the loops corresponding to cycles in the graph $\mathcal{R}_{A}$. Note that knowing these numbers is enough to calculate all the invariants of $\eta$.

Indeed, a cycle in the graph $\mathcal{R}_{A}$ is an alternating sequence $\ldots v_{i} f_{i} v_{i+1} f_{i+1} \ldots$ of vertices and forbidden threads in $Q$. Since $\eta$ is transverse to each arc $L_{v_{i}}$, we can calculate the winding number as the sum of winding numbers of the segments of the cycle connecting a point in $L_{v_{i}}$ with a point in $L_{v_{i+1}}$ through the disk $D_{f_{i}}$. Recall that the boundary of this disk is formed by the arcs $L_{v}$ where $v$ runs through vertices in the thread $f_{i}$, and the parts of the boundary labeled by arrows in $f_{i}$ (as in Fig. 2). Now we claim that the contribution to the winding number from the segment $v_{i} f_{i} v_{i+1}$ is equal to

$$
w_{\eta}\left(v_{i} f_{i} v_{i+1}\right)= \begin{cases}1-m+\sum_{j=1}^{m} \operatorname{deg}\left(\alpha_{j}\right), & v_{i} \stackrel{\alpha_{1}}{\rightarrow} \cdots \stackrel{\alpha_{m}}{\longrightarrow} v_{i+1} \subset f_{i} \\ -1+m-\sum_{j=1}^{m} \operatorname{deg}\left(\beta_{j}\right), & v_{i+1} \stackrel{\beta_{1}}{\longrightarrow} \cdots \stackrel{\beta_{m}}{\longrightarrow} v_{i} \subset f_{i}\end{cases}
$$


Indeed, this follows immediately by looking at the polygon formed by the $\operatorname{arcs} L_{v_{i}}$ and $L_{v_{i+1}}$, by the segment of our cycle between them, and by the part of the boundary of $D_{f_{i}}$ between these arcs.

\subsection{Application to finite-dimensional gentle algebras and examples}

It is well known that gentle algebras are Koszul and that the Koszul dual of a gentle algebra is again gentle, corresponding to the dual combinatorial data (see [9, Sec. 3.3] where what we call "gentle" is called "locally gentle"). Furthermore, under this duality homologically smooth graded gentle algebras are exchanged with finite-dimensional ones. Thus, using Koszul duality we can convert our results into those about finitedimensional gentle algebras.

Let $A$ be a finite-dimensional gentle algebra with grading in degree 0 . Let $A^{!}$be the Koszul dual gentle algebra (with respect to the generators given by the edges). We equip $A^{!}$with the grading for which all edges have degree 1 (i.e., path-length grading). Then the result of Keller in [19, Sec. 10.5] ("exterior" case) gives an equivalence

$$
D_{f}(A) \simeq D\left(A^{!}\right)
$$

where $D_{f}(A)$ is the bounded derived category of finite-dimensional $A$-modules (and $D\left(A^{!}\right)$is the perfect derived category of $A^{!}$viewed as a dg-algebra, as before).

Furthermore, it is easy to check that the AAG-invariants of $A$ and $A^{!}$are the same. Thus, Corollary 3.14 leads to the following result.

Corollary 3.16 Let $A$ and $B$ be finite-dimensional gentle algebras with grading in degree 0 , such that the AAG-invariants of $A$ and $B$ coincide (up to permutation) and satisfy one of the conditions (a)-(c) of Corollary 3.14. Then

$$
D_{f}(A) \simeq D_{f}(B)
$$

Example 3.17 Here is an example illustrating the construction of associating a surface to a gentle algebra. Consider the gentle algebra given in Fig. 3.

The forbidden threads are given by $\left\{a, b d, c, e_{4}\right\}$. The permitted threads are given by $\left\{c b a, d, e_{3}, e_{4}\right\}$. The combinatorial boundary components are given by $\left\{p_{3} f_{3} p_{2} f_{2} p_{1} f_{1}, p_{4} f_{4}\right\}$ where, $f_{1}=e_{4}, p_{1}=e_{4}, f_{2}=c, p_{2}=e_{3}, f_{3}=b d$, $p_{3}=c b a$, and $f_{4}=a, p_{4}=d$.

The associated ribbon graph is given in Fig. 4, where the cyclic order at vertices are given by counter-clockwise rotation.

Figure 5 depicts the corresponding surface, together with the dual $\operatorname{arcs} L_{1}, L_{2}, L_{3}, L_{4}$.

As this is a genus zero surface, the line field is determined by the winding numbers along the boundary components. The winding number along the interior puncture

Fig. 3 An example of gentle algebra

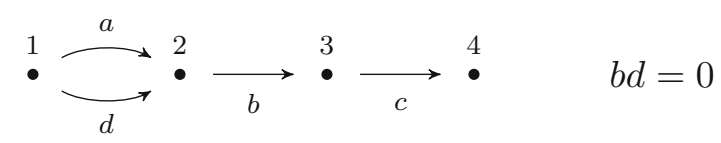


Fig. 4 Ribbon graph associated to a gentle algebra

Fig. 5 Surface associated to a gentle algebra

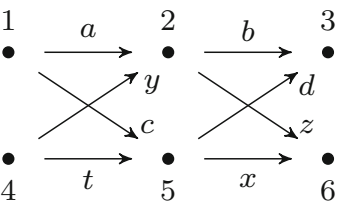

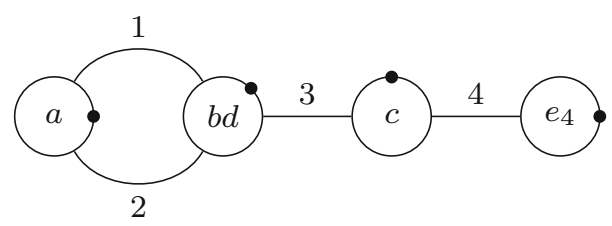

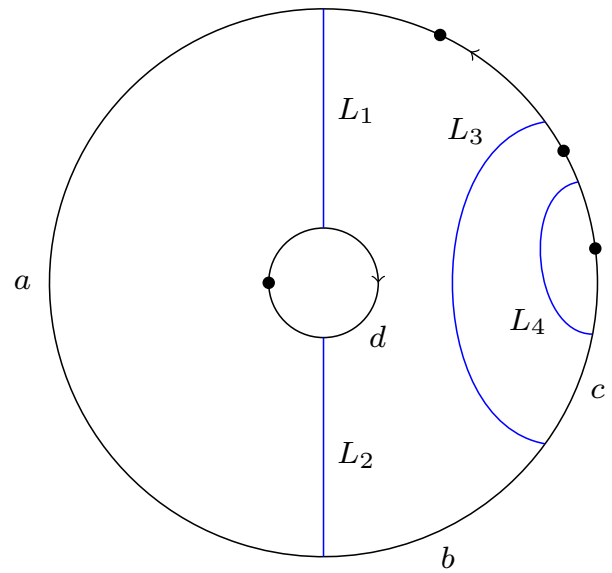

$$
z a=b y=x c=d t=0
$$

Fig. 6 Another example of a gentle algebra

which corresponds to the combinatorial boundary component $p_{4} f_{4}$ is given by $|a|-|d|$ and the winding number along the outer boundary component which corresponds to the combinatorial boundary component $p_{3} f_{3} p_{2} f_{2} p_{1} f_{1}$ is the negative of this (since the two boundary components are homotopic but oriented in an opposite way) but can also be computed as $(-|a|-|b|-|c|)+(|b|+|d|-1)+|c|+1=|d|-|a|$.

Example 3.18 Here is another example that produces a genus 1 surface with 2 boundary components. Consider the gentle algebra given by Fig. 6 .

The forbidden threads are given by $\{z a, b y, x c, d t\}$, and the permitted threads are given by $\{b a, d c, x t, z y\}$. The combinatorial boundary components are given by $\left\{p_{2} f_{2} p_{1} f_{1}, p_{4} f_{4} p_{3} f_{3}\right\}$ where $f_{1}=d t, p_{1}=z y, f_{2}=x c, p_{2}=b a$, and $f_{3}=z a, p_{3}=d c, f_{4}=b y, p_{4}=x t$.

The corresponding surface is given in Fig. 7.

This is a genus 1 surface with 2 boundary components. To determine the line field we need to compute its winding number along the booundary components corresponding to $b_{1}=p_{2} f_{2} p_{1} f_{1}$ and $b_{2}=p_{4} f_{4} p_{3} f_{3}$ as well as winding numbers along non-separating curves depicted in grey. The horizontal one corresponds 


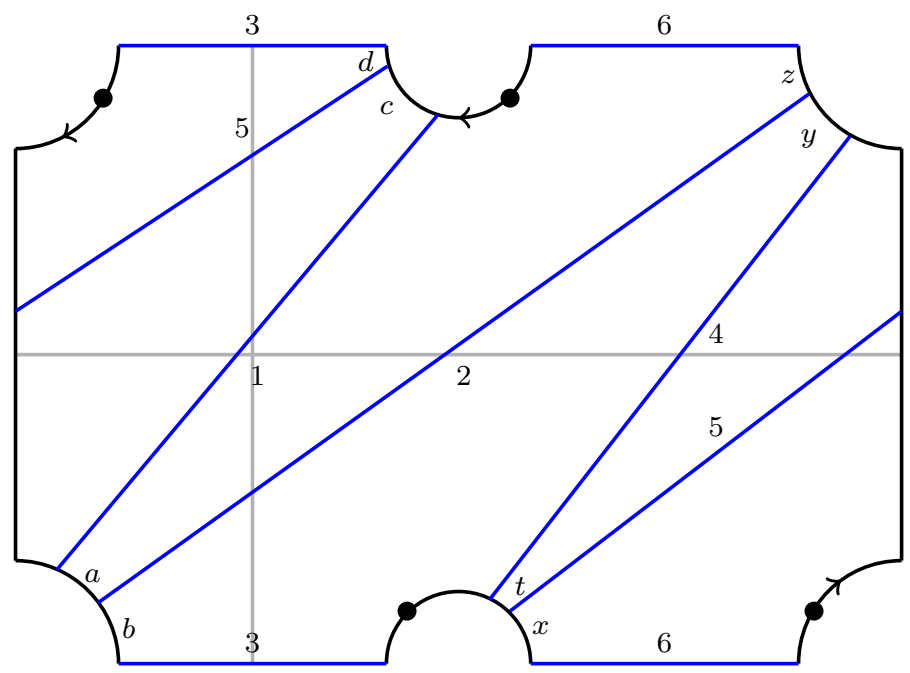

Fig. 7 Genus 1 surface with 2 boundary components. Left-right and top-bottom are identified

to the cycle $\alpha=f_{2} v_{5} f_{1} v_{4} f_{4} v_{2} f_{3} v_{1}$, and the vertical one corresponds to the cycle $\beta=f_{1} v_{5} f_{2} v_{1} f_{3} v_{2} f_{4} v_{3}$. From the formulae given, it is easy to compute

$$
\begin{aligned}
w_{\eta}\left(b_{1}\right) & =-|a|-|b|+(|x|+|c|-1)-|z|-|y|+(|d|+|t|-1) \\
w_{\eta}\left(b_{2}\right) & =-|t|-|x|+(|y|+|b|-1)-|c|-|d|+(|z|+|a|-1) \\
w_{\eta}(\alpha) & =|t|-|y|+|a|-|c| \\
w_{\eta}(\beta) & =-|b|-|a|+|c|+|d|
\end{aligned}
$$

Remark 3.19 An optimist's conjecture would be that conversely if $A$ and $B$ are homologically smooth graded gentle algebras which are derived equivalent, then there exists a homeomorphism $\phi: \Sigma_{A} \rightarrow \Sigma_{B}$ inducing a bijection $\Lambda_{A} \rightarrow \Lambda_{B}$ and such that $\phi_{*}\left(\eta_{A}\right)$ is homotopic to $\eta_{B}$. Note that to prove this, one needs to show that the topological type of $\left(\Sigma_{A}, \Lambda_{A} ; \eta_{A}\right)$ is a derived invariant of $A$. This is encoded by the numerical invariants of $\eta_{A}$ introduced in Theorem 1.8 (from which one can recover the topological type of the surface), together with the numbers of marked points on each boundary component.

Remark 3.20 In Theorem 3.11, it is possible to drop the assumption that $A$ is smooth. Assume for simplicity that $A$ is proper. In this case, the surface $\Sigma$ would be glued together from the disks $D_{f}$ associated to forbidden threads as before, and also disks $D_{c}$ with an interior hole, associated with forbidden cycles. In other words, $D_{c}$ is an annulus whose inner boundary component has no marked points and is not glued to anything, while its outer boundary component is connected by strips, corresponding to the vertices in $c$, to other disks (this boundary component of $D_{c}$ still has no stops). In the presence of unmarked boundary components, there is a dual construction to the construction of partially wrapped Fukaya categories, $\mathcal{W}(\Sigma, \Lambda ; \eta)$, namely, the 
infinitesimal wrapped Fukaya categories $\mathcal{F}(\Sigma, \Lambda ; \eta)$, originally introduced for general symplectic manifolds in [22] and studied in the case of surfaces in [21]. Its objects are graded Lagrangians which do not end on the unmarked components of the boundary. Thus, for non-smooth proper gentle algebras, a version of Theorem 3.11 should state the equivalence

$$
D(A) \simeq \mathcal{F}\left(\Sigma_{A}, \Lambda_{A} ; \eta_{A}\right)
$$

However, we have not checked that the collection of Lagrangians $\left\{L_{v}\right\}$ given by the construction in Theorem 3.11 (and modified as above) generates $\mathcal{F}\left(\Sigma_{A}, \Lambda_{A} ; \eta_{A}\right)$.

Remark 3.21 We note that the statement of Theorem 3.11 is mentioned in Section 3.4 of [15]. In the special case when the gentle algebra is trivially graded, the construction of the surface $\Sigma$ and the dual set of Lagrangians to $\left\{L_{i}\right\}$ appeared again in [23] after this work was posted on arXiv. The authors of [23] work with the Kozsul dual gentle algebra from a representation theoretical perspective. From the point of view of [15], these Koszul dual algebras can be understood as the infinitesimal Fukaya categories as explained in the previous remark. Note also that when every boundary component has at least one stop which is equivalent to requiring that corresponding gentle algebras are homologically smooth and proper, infinitesimal and partially wrapped Fukaya categories are equivalent. We refer to [13] for general results about Koszul duality in the setting of Fukaya categories.

\section{Derived equivalences between stacky curves}

\subsection{Chains}

Recall that in [21] we considered stacky curves $C\left(r_{0}, \ldots, r_{n} ; k_{1}, \ldots, k_{n-1}\right)$ obtained by gluing weighted projective lines

$$
B\left(r_{0}, r_{1}\right), B\left(r_{1}, r_{2}\right), \ldots, B\left(r_{n-1}, r_{n}\right)
$$

into a chain, where $k_{i} \in\left(\mathbb{Z} / r_{i}\right)^{*}$ are used to determine the stacky structure of the nodes in this chain.

Here $B(a, b)$, for $a, b>0$, denotes the weighted projective line stack $\left(\mathbb{A}^{2} \backslash 0\right) / \mathbb{G}_{m}$, where $\mathbb{G}_{m}$ acts with weights $(a, b)$ (see e.g., [6, Sec. 2] and references therein). It has two stacky points $q_{-}$and $q_{+}$such that $\operatorname{Aut}\left(q_{-}\right)=\mu_{a}$, Aut $\left(q_{+}\right)=\mu_{b}$. To form the chain $C\left(r_{0}, \ldots, r_{n} ; k_{1}, \ldots, k_{n-1}\right)$, we glue the point $q_{+}$in $B\left(r_{i-1}, r_{i}\right)$ with the point $q_{-}$in $B\left(r_{i}, r_{i+1}\right)$, so that the obtained stacky node locally looks like the quotient of $x y=0$ by the action of $\mu_{r_{i}}$ of the form $\zeta \cdot(x, y)=\left(\zeta^{k_{i}} x, \zeta y\right)$.

Note that in [29] similar stacky curves are considered but with all $k_{i}=-1$ (the corresponding stacky nodes are called balanced).

We also allow the possibility for $r_{0}=0$ (resp., $\left.r_{n}=0\right)$ ): in this case $B\left(0, r_{1}\right)$ (resp. $\left.B\left(r_{n-1}, 0\right)\right)$ denotes the weighted affine line $\mathbb{A}^{1}\left(r_{1}\right)=B\left(1, r_{1}\right) \backslash\left\{q_{-}\right\}$(resp. $\left.\mathbb{A}^{1}\left(r_{n-1}\right)=B\left(r_{n-1}, 1\right) \backslash\left\{q_{+}\right\}\right)$. 


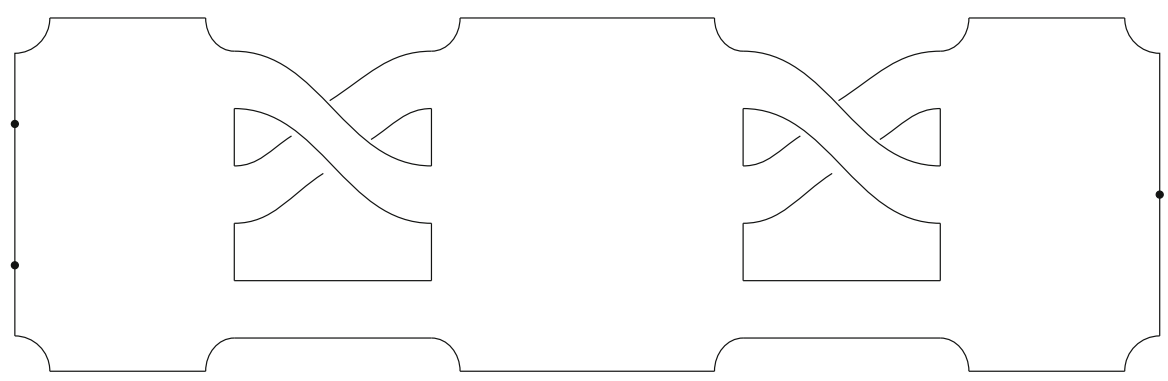

Fig. 8 Surface glued from annuli (top and bottom are identified). $\left(r_{0}, r_{1}, r_{2}, r_{3}\right)=(2,3,3,1), \sigma_{1}=\sigma_{2}$ : $(1,2,3) \rightarrow(2,1,3)$

We showed in [21, Thm. B] that the bounded derived category of coherent sheaves on such a stacky curve is equivalent to the partially wrapped Fukaya category of a surface obtained by a certain gluing of the annuli that we will now describe.

Namely, let $A\left(r, r^{\prime}\right)$ denote the annulus with ordered boundary components that has $r$ marked points $p_{1}^{-}, \ldots, p_{r}^{-}$on the first component and $r^{\prime}$ marked points $p_{1}^{+}, \ldots, p_{r^{\prime}}^{+}$ on the second boundary component. We visualize $A\left(r, r^{\prime}\right)$ as a rectangle with upper and lower sides glued, the left side containing the points $p_{i}^{-}$and the right side containing the points $p_{i}^{+}$, where the points are ordered vertically (the index increases when moving up).

Given a collection of permutations $\sigma_{i} \in \mathfrak{S}_{r_{i}}, i=1, \ldots, n-1$, we consider the surface $\Sigma^{\operatorname{lin}}\left(r_{0}, \ldots, r_{n} ; \sigma_{1}, \ldots, \sigma_{n-1}\right)$ obtained by gluing the annuli

$$
A\left(r_{0}, r_{1}\right), A\left(r_{1}, r_{2}\right), \ldots, A\left(r_{n-1}, r_{n}\right)
$$

in the following way ("lin" stands for "linear", since we place annuli in a line). For each $i=1, \ldots, n-1, j=1, \ldots, r_{i}$, we glue a small segment of the boundary around the marked point $p_{j}^{+}$in $A\left(r_{i-1}, r_{i}\right)$ with a small segment of the boundary around the point $p_{\sigma_{i}(j)}^{-}$in $A\left(r_{i}, r_{i+1}\right)$ by attaching a strip, as in Fig. 8.

Note that the resulting surface has two special boundary components equipped with $r_{0}$ and $r_{n}$ marked points, respectively (there are no other marked points on the other boundary components). There are also other boundary components that arise in the process of gluing. Namely, for each $i=1, \ldots, n-1$, the boundary components situated between the $i$ th and the $(i+1)$ st annuli are in bijection with cycles in the cycle decomposition of the commutator $\left[\sigma_{i}, \tau\right] \in \mathfrak{S}_{r_{i}}$, where $\tau$ is the cyclic permutation $j \mapsto j-1$.

We equip $\Sigma^{\text {lin }}\left(r_{0}, \ldots, r_{n} ; \sigma_{1}, \ldots, \sigma_{n-1}\right)$ with a line field $\eta$ that corresponds to the horizontal direction in Fig. 8. Note that its restriction to each annulus is the standard line field that has zero winding numbers on both boundary components (this is the same choice of a line field that was made in [21, Sec. 2]).

It is easy to see that the winding numbers of $\eta$ on boundary components are given as follows. For the two special boundary components the winding numbers are equal to zero. For a boundary component corresponding to a $k$-cycle in the cycle decomposition of $\left[\sigma_{i}, \tau\right]$, the winding number is $-2 k$. 
We are going to prove that in fact all winding numbers associated with $\eta$ are even. For this it is useful to construct a graph

$$
\Gamma\left(r_{1}, \ldots, r_{n-1}\right) \subset \Sigma^{l i n}\left(r_{0}, \ldots, r_{n} ; \sigma_{1}, \ldots, \sigma_{n-1}\right),
$$

which is a homotopy retract of the surface. Namely, we take one vertex in the interior of each annulus: this gives us $n$ vertices $v_{1}, \ldots, v_{n}$. Then we add a loop $\gamma_{i}$ at each $v_{i}$, corresponding to the vertical circle in the $i$ th annulus. Then for each of the $r_{i}$ strips connecting the $i$ th annulus with the $(i+1)$ st we add an edge from $v_{i}$ to $v_{i+1}$.

Lemma 4.1 One has $\left[w_{\eta}\right]^{(2)}=0$, i.e., all winding numbers of $\eta$ are even.

Proof The embedding of the graph $\Gamma\left(r_{1}, \ldots, r_{n-1}\right)$ into $\Sigma^{l i n}\left(r_{0}, \ldots, r_{n} ; \sigma_{1}, \ldots, \sigma_{n-1}\right)$ induces an isomorphism on homology. Hence, $H_{1}\left(\Sigma^{l i n}\left(r_{0}, \ldots, r_{n} ; \sigma_{1}, \ldots, \sigma_{n-1}\right)\right)$ is spanned by the loops $\gamma_{i}$ together with the loops formed by pairs of edges connecting $v_{i}$ with $v_{i+1}$. The latter loops can have plane projections of one of the two types: they look either like circles or like figure eight curves, depending on whether the projections of the corresponding edges cross or not. The winding number of $\eta$ along a circle in the plane is -2 , while the winding number along a figure eight curve is 0 . Since $\eta$ is constant along vertical lines, its winding numbers along $\gamma_{i}$ are 0 . Now the result follows from the fact that $\left[w_{\eta}\right]^{(2)}$ is a homomorphism.

To get the surface related to the stacky curve $C\left(r_{0}, \ldots, r_{n} ; k_{1}, \ldots, k_{n-1}\right)$, we now take permutations $\sigma_{i}$ of a special kind. Namely, for each $i=1, \ldots, n-1$, we consider the permutation

$$
\sigma_{i}: x \mapsto-k_{i} x
$$

of $\mathbb{Z} / r_{i} \mathbb{Z}$. We denote the resulting surface by $\Sigma^{l i n}\left(r_{0}, \ldots, r_{n} ; k_{1}, \ldots, k_{n-1}\right)$. We equip it with $r_{0}$ and $r_{n}$ stops on two special boundary components, and denote this set of stops as $\Lambda_{r_{0}, r_{n}}$. Now [21, Thm. B] states that

$D^{b}\left(\operatorname{Coh} C\left(r_{0}, \ldots, r_{n} ; k_{1}, \ldots, k_{n-1}\right)\right) \cong \mathcal{W}\left(\Sigma^{l i n}\left(r_{0}, \ldots, r_{n} ; k_{1}, \ldots, k_{n-1}\right), \Lambda_{r_{0}, r_{n}} ; \eta\right)$.

For example, taking $r_{0}=r_{n}=0$, which corresponds to replacing the first and last weighted projective line by weighted affine lines, we will get the fully wrapped Fukaya categories (with no stops).

Note that for each $i$ the commutator $\left[\sigma_{i}, \tau\right]$ is given by $x \mapsto x+k_{i}+1 \bmod \left(r_{i}\right)$, so its cycle decomposition has $p_{i}=\operatorname{gcd}\left(k_{i}+1, r_{i}\right)$ cycles of length $r_{i} / p_{i}$. Thus, the boundary winding numbers of $\eta$ on $\Sigma^{l i n}\left(r_{0}, \ldots, r_{n} ; k_{1}, \ldots, k_{n-1}\right)$ are

- 0 on each of the two special boundary components (that have marked points);

- for each $i=1, \ldots, n-1$, the winding number $-2 r_{i} / p_{i}$ repeated $p_{i}$ times.

The genus of the surface $\Sigma^{l i n}\left(r_{0}, \ldots, r_{n} ; k_{1}, \ldots, k_{n-1}\right)$ is given by

$$
g=\frac{1}{2} \sum_{i=1}^{n-1}\left(r_{i}-p_{i}\right) .
$$


Now we are going to apply Corollary 1.10 to construct examples of different data $\left(r_{0}, \ldots, r_{n} ; k_{1}, \ldots, k_{n-1}\right)$ that lead to surfaces which are homeomorphic in a way preserving the marked points on boundary components and the line fields. This will give equivalences between corresponding partially wrapped Fukaya categories and hence between the corresponding derived categories of stacky curves.

Theorem 4.2 The graded surface with stops $\Sigma^{\text {lin }}\left(r_{0}, \ldots, r_{n} ; k_{1}, \ldots, k_{n-1}\right)$ is determined up to a graded homeomorphism by the unordered pair of numbers $\left(r_{0}, r_{n}\right)$ and by the unordered collection of numbers

$$
\left(\left(r_{1} / p_{1}\right)^{p_{1}}, \ldots,\left(r_{n-1} / p_{n-1}\right)^{p_{n-1}}\right),
$$

where $\left(r_{i} / p_{i}\right)^{p_{i}}$ denotes the number $r_{i} / p_{i}$ repeated $p_{i}$ times. Hence, the same data determines the category $D^{b}\left(\operatorname{Coh} C\left(r_{0}, \ldots, r_{n} ; k_{1}, \ldots, k_{n-1}\right)\right)$ up to equivalence.

Proof The two special components (that have stops on them) are the only ones that have the winding number 0 . The winding numbers of all the other boundary components are determined by the sequence (4.2). Thus, our claim is that our graded surfaces are determined by their boundary invariants (numbers of points on components and winding numbers). We want to deduce this from Corollary 1.10 .

In the case when genus is 0 , there is nothing more to check. In the case when genus is $\geq 2$, we observe that by Lemma 4.1, the invariant $\sigma$ vanishes for our line field $\eta$. On the other hand, because of the two special components with the winding number 0 , the Arf-invariant does not appear, so we are done in this case.

Finally, if the surface $\Sigma^{l i n}\left(r_{0}, \ldots, r_{n} ; k_{1}, \ldots, k_{n-1}\right)$ has genus 1 then we claim that $\widetilde{A}(\eta)=2$. Indeed, by Lemma 4.1, $\widetilde{A}(\eta)$ is even, so this follows from the existence of a boundary component with the winding number 0 .

\subsubsection{Merging stacky nodes into one}

Note that if $k_{i}=-1$ for some $i$ (which means that the corresponding node on the stacky curve is balanced) then gluing of $A\left(r_{i-1}, r_{i}\right)$ with $A\left(r_{i}, r_{i+1}\right)$ results in $r_{i}$ boundary components on which $\eta$ has the winding number -2 . Thus, if $I \subset[1, n-1]$ is a subset of indices $i$ such that $k_{i}=-1$, then setting $r_{I}=\sum_{i \in I} r_{i}$, we get a graded homeomorphism

$$
\Sigma^{l i n}\left(r_{0}, \ldots, r_{n} ; k_{1}, \ldots, k_{n-1}\right) \simeq \Sigma^{l i n}\left(r_{0}, r_{I},\left(r_{i}\right)_{i \notin I}, r_{n} ;-1,\left(k_{i}\right)_{i \notin I}\right) .
$$

Corollary 4.3 Let $I \subset[1, n-1]$ is a subset such that $k_{i}=-1$ for $i \in I$, and let $r_{I}=\sum_{i \in I} r_{i}$. Then there is an equivalence

$D^{b}\left(\operatorname{Coh} C\left(r_{0}, \ldots, r_{n} ; k_{1}, \ldots, k_{n-1}\right)\right) \simeq D^{b}\left(\operatorname{Coh} C\left(r_{0}, r_{I},\left(r_{i}\right)_{i \notin I}, r_{n} ;-1,\left(k_{i}\right)_{i \notin I}\right)\right)$.

In the particular case $I=[1, n-1]$ (corresponding to surfaces of genus 0 ), the derived equivalence of the above Corollary,

$$
D^{b}\left(\operatorname{Coh} C\left(r_{0}, \ldots, r_{n} ;-1, \ldots,-1\right)\right) \cong D^{b}\left(\operatorname{Coh} C\left(r_{0}, r_{1}+\ldots+r_{n-1}, r_{n} ;-1\right)\right),
$$

was proved in [28]. 
Note that the surfaces $\Sigma^{l i n}\left(r_{\bullet} ; k_{\bullet}\right)$ can have genus 1 only when $r_{i_{0}}-p_{i_{0}}=2$ for some $i_{0} \in[1, n-1]$ and $r_{i}=p_{i}$ for $i \neq i_{0}$. This can happen only when either $r_{i_{0}}=3$ or $r_{i_{0}}=4$ and $k_{i_{0}}=1$. These cases are distinguished by the presence of the boundary components with the winding number either -6 or -4 . So in the cases when the genus is 0 or 1 we do not get any other derived equivalences between our stacky chain curves except those due to merging of balanced nodes.

In higher genus we can sometimes merge unbalanced nodes as well. For example, if $\operatorname{gcd}(k+1, r)=1$ then for any divisor $d$ of $k+1, d$ stacky nodes of type $(r ; k)$ can be merged into one stacky node of type $(d r ; k)$.

Corollary 4.4 Assume that for $k \in \mathbb{Z}_{r}^{*}$ one has $\operatorname{gcd}(k+1, r)=1$, and let $d>0$ be a divisor of $k+1$. Then we have an equivalence

$$
\begin{aligned}
& D^{b}\left(\operatorname{Coh} C\left(r_{0},(r)^{d}, r_{d+1}, \ldots, r_{n} ;(k)^{d}, k_{d+1}, \ldots, k_{n-1}\right)\right) \\
& \simeq D^{b}\left(\operatorname{Coh} C\left(r_{0}, d r, r_{d+1}, \ldots, r_{n} ; k, k_{d+1}, \ldots, k_{n-1}\right)\right)
\end{aligned}
$$

Proof We have $\operatorname{gcd}(k+1, d r)=d \cdot \operatorname{gcd}\left(\frac{k+1}{d}, r\right)=d$. Thus, the pair $(d r ; k)$ contributes $d p$ boundary components with the winding number $-2 r$, which is the same as the contribution of $d$ pairs $(r ; k)$.

\subsubsection{Derived equivalent quotients of the coordinate cross}

To get a more interesting derived equivalence in the case of genus $\geq 2$, let us specialize to the case $n=2, r_{0}=r_{2}=0, r_{1}=r$. Note that the corresponding stacky curve $C(0, r, 0 ; k)$ is the global quotient of the affine coordinate cross $x y=0$ by the $\mu_{r^{-}}$ action $\zeta \cdot(x, y)=\left(\zeta^{k} x, \zeta y\right)$. We obtain the following derived equivalences between these affine stacky curves.

Corollary 4.5 For $k, k^{\prime} \in(\mathbb{Z} / r)^{*}$, such that $\operatorname{gcd}(k+1, r)=\operatorname{gcd}\left(k^{\prime}+1, r\right)$, there exists an equivalence

$$
D^{b} \operatorname{Coh}(C(0, r, 0 ; k)) \simeq D^{b} \operatorname{Coh}\left(C\left(0, r, 0 ; k^{\prime}\right)\right)
$$

Note that if $k \cdot k^{\prime} \equiv 1 \bmod r$ then we have an isomorphism $C(0, r, 0 ; k) \simeq$ $C\left(0, r, 0 ; k^{\prime}\right)$ induced by the involution $(x, y) \mapsto(y, x)$ on the coordinate cross. The simplest example of a nontrivial derived equivalence of this kind is when $r=5$, $k=1$ and $k^{\prime}=2$. It would be interesting to explain this derived equivalence in a purely algebro-geometric way. Our guess is that this can be done using the variation of GIT quotient technique.

\subsection{Rings}

Now let us consider another class of stacky curves considered in [21], denoted by $R\left(r_{1}, \ldots, r_{n} ; k_{1}, \ldots, k_{n}\right)$. They are defined by gluing the weighted projective lines $B\left(r_{1}, r_{2}\right), B\left(r_{2}, r_{3}\right), \ldots, B\left(r_{n}, r_{1}\right)$ into a ring, where as before $k_{i} \in\left(\mathbb{Z} / r_{i}\right)^{*}$ are used 
to determine the stacky structure of the nodes. Thus, the point $q_{+}$in $B\left(r_{i-1}, r_{i}\right)$ is glued with the point $q_{-}$in $B\left(r_{i}, r_{i+1}\right)$ for all $i \in \mathbb{Z} / n$.

On the symplectic side we modify our definition of the surfaces $\Sigma^{l i n}\left(r_{0}, \ldots, r_{n} ; \sigma_{1}\right.$, $\left.\ldots, \sigma_{n-1}\right)$ as follows. Starting with the annuli $A\left(r_{1}, r_{2}\right), A\left(r_{2}, r_{3}\right), \ldots, A\left(r_{n}, r_{1}\right)$ we now glue them circularly using permutations $\sigma_{1}, \ldots, \sigma_{n}$, so that $A\left(r_{i-1}, r_{i}\right)$ is connected by $r_{i}$ strips with $A\left(r_{i}, r_{i+1}\right)$, for $i \in \mathbb{Z} / n$. Thus, the corresponding surface could be represented similarly to Fig. 8 but with the right and left ends identified (so that the corresponding boundary components disappear). We denote the resulting surface by $\Sigma^{c i r}\left(r_{1}, \ldots, r_{n} ; \sigma_{1}, \ldots, \sigma_{n}\right)$.

Similarly to the case of a linear gluing it is equipped with a natural line field $\eta$ that corresponds to the horizontal direction when the surface is depicted as on Fig. 8. As before, the winding numbers of $\eta$ on the boundary component corresponding to a $k$-cycle in $\left[\sigma_{i}, \tau\right]$ is equal to $-2 k$.

The analog of the graph $\Gamma\left(r_{1}, \ldots, r_{n-1}\right)$ for circular gluing is given by the graph

$$
\Gamma^{c i r}\left(r_{1}, \ldots, r_{n}\right) \subset \Sigma^{c i r}\left(r_{1}, \ldots, r_{n} ; \sigma_{1}, \ldots, \sigma_{n}\right)
$$

that still has $n$ vertices $v_{1}, \ldots, v_{n}$, a loop $\gamma_{i}$ at each $v_{i}$, and $r_{i}$ edges connecting $v_{i}$ to $v_{i+1}$, for $i \in \mathbb{Z} / n$. This graph is a homotopy retract of the surface, so we can calculate the homology just by analyzing loops in $\Gamma^{c i r}\left(r_{1}, \ldots, r_{n}\right)$. In particular, we see that the homology is spanned by the loops $\gamma_{i}$, the loops formed by pairs of edges connecting $v_{i}$ with $v_{i+1}$, and by one more loop $\beta$ corresponding to a horizontal line in Fig. 8 The analog of Lemma 4.1 still holds in this case and is proved similarly: all winding numbers of $\eta$ are even. Note that winding number along $\beta$ is zero since $\eta$ is constant along horizontal lines.

As before, we specialize to the case of permutations of the form (4.1) and denote the corresponding surface by $\Sigma^{c i r}\left(r_{1}, \ldots, r_{n} ; k_{1}, \ldots, k_{n}\right)$. The boundary winding numbers of $\eta$ on this surface are calculated as before (but now we do not have two special boundary components). The genus of this surface is given by

$$
g=1+\frac{1}{2} \sum_{i=1}^{n}\left(r_{i}-p_{i}\right)
$$

where $p_{i}=\operatorname{gcd}\left(k_{i}+1, r_{i}\right)$.

By $[21$, Thm. B], we have an equivalence

$$
D^{b}\left(\operatorname{Coh} R\left(r_{1}, \ldots, r_{n} ; k_{1}, \ldots, k_{n}\right)\right) \cong \mathcal{W}\left(\Sigma^{c i r}\left(r_{1}, \ldots, r_{n} ; k_{1}, \ldots, k_{n}\right) ; \eta\right) .
$$

As before, we can use Corollary 1.10 to get derived equivalences between the corresponding stacky curves.

We have $\sigma(\eta)=0$ for our line field, so in the case when $r_{i} / p_{i}$ is odd for all $i$, the corresponding quadratic form $\bar{q}_{\eta}$ on $\mathbb{Z}_{2}^{2 g}$ is well defined and we have to calculate its Arf-invariant. 
Definition 4.6 For a permutation $\sigma \in S_{d}$, let us consider the vector space $V(\sigma)$ over $\mathbb{Z}_{2}$ with the basis $\alpha_{1}, \ldots, \alpha_{d}$, the even pairing such that $\alpha_{i} \cdot \alpha_{j}$ for $i<j$ is given by

$$
\alpha_{i} \cdot \alpha_{j}= \begin{cases}0 & \sigma(i)<\sigma(j), \\ 1 & \sigma(i)>\sigma(j),\end{cases}
$$

and the unique quadratic form $q_{\sigma}$ compatible with this pairing such that $q_{\sigma}\left(\alpha_{i}\right)=0$ for all $i$. Let $\bar{V}(\sigma)$ be the quotient of $V(\sigma)$ by the kernel of the pairing. If the restriction of $q_{\sigma}$ to the kernel of the pairing is zero then $q_{\sigma}$ descends to a quadratic form $\bar{q}_{\sigma}$ on $\bar{V}(\sigma)$.

Lemma 4.7 (i) For $k \in \mathbb{Z}_{r}^{*}$, let us consider the permutation $\sigma_{r}(k)$ of $\mathbb{Z}_{r} \backslash\{0\}=$ $\{1, \ldots, r-1\}$ given by $x \mapsto-k x$. Then the corresponding quadratic form $q(r, k):=q_{\sigma_{r}(k)}$ is trivial on the kernel of the pairing on $V\left(\sigma_{r}(k)\right)$ if and only if $r / p$ is odd, where $p=\operatorname{gcd}(k+1, r)$. If this is the case then $q(r, k)$ descends to a nondegenerate quadratic form $\bar{q}(r, k)$ on the $(r-p)$-dimensional space $\bar{V}\left(\sigma_{r}(k)\right)$.

(ii) Consider the standard line field $\eta$ on the surface $\Sigma^{\text {cir }}\left(r_{1}, \ldots, r_{n} ; k_{1}, \ldots, k_{n}\right)$. Assume that all $r_{i} / p_{i}$ are odd, where $p_{i}=\operatorname{gcd}\left(k_{i}+1, r_{i}\right)$. Then the quadratic form $\bar{q}_{\eta}$ is well defined, and is the direct sum of $\bar{q}\left(r_{i}, k_{i}\right)$ over $i=1, \ldots, n$, and the form $x^{2}+y^{2}+x y$ on $\mathbb{Z}_{2} \oplus \mathbb{Z}_{2}$. Hence, in this case

$$
A(\eta)=\sum_{i=1}^{n} A\left(\bar{q}\left(r_{i}, k_{i}\right)\right)+1 \bmod (2) .
$$

Proof We are going to study the quadratic form associated with the the surface $\Sigma=$ $\Sigma^{c i r}(r ; k)$. Let us look at the simple curves $\alpha_{i}, i=1, \ldots, r-1$, on $\Sigma$, depicted on Fig. 9. In addition, we have two simple curves, $\beta$ and $\gamma$, corresponding to a horizontal and a vertical line on Fig. 9.

Using the graph $\Gamma^{c i r}(r)$, we see that the classes $[\beta],[\gamma]$ and $\left(\left[\alpha_{i}\right]\right)_{i=1, \ldots, r-1}$ span $H_{1}\left(\Sigma, \mathbb{Z}_{2}\right)$. Furthermore, the restriction of the intersection pairing to the subspace generated by $\left(\left[\alpha_{i}\right]\right)$ gives precisely the pairing on $V\left(\sigma_{r}(k)\right)$. On the other hand, both $[\beta]$ and $[\gamma]$ are orthogonal to this subspace and $\beta \cdot \gamma=1$. It follows that the kernel of the intersection pairing on $H_{1}\left(\Sigma, \mathbb{Z}_{2}\right)$ is equal to the kernel of the pairing on $V\left(\sigma_{r}(k)\right)$, and the quotient of $H_{1}\left(\Sigma, \mathbb{Z}_{2}\right)$ by this kernel is the direct sum of $\bar{V}\left(\sigma_{r}(k)\right)$ and the 2dimensional space spanned by $[\beta]$ and $[\gamma]$. Since the kernel of the intersection pairing is spanned by the classes of the $p$ boundary components and is $(p-1)$-dimensional, we deduce that $\operatorname{dim} \bar{V}\left(\sigma_{r}(k)\right)=r-p$.

Furthermore, the winding number along each $\alpha_{i}$ is -2 so $q_{\eta}\left(\alpha_{i}\right)=0 \bmod (4)$. Thus, the restriction of the $\mathbb{Z}_{2}$-valued form $q_{\eta} / 2$ to the subspace $V\left(\sigma_{r}(k)\right)$ is precisely $q(r, k)$. It follows that $q(r, k)$ vanishes on the kernel of the pairing if and only if $q_{\eta}$ vanishes on the boundary cycles, which happens exactly when $r / p$ is odd (recall that the value of $q_{\eta}$ on any boundary cycle is $\left.2-2 r / p \bmod (4)\right)$. 


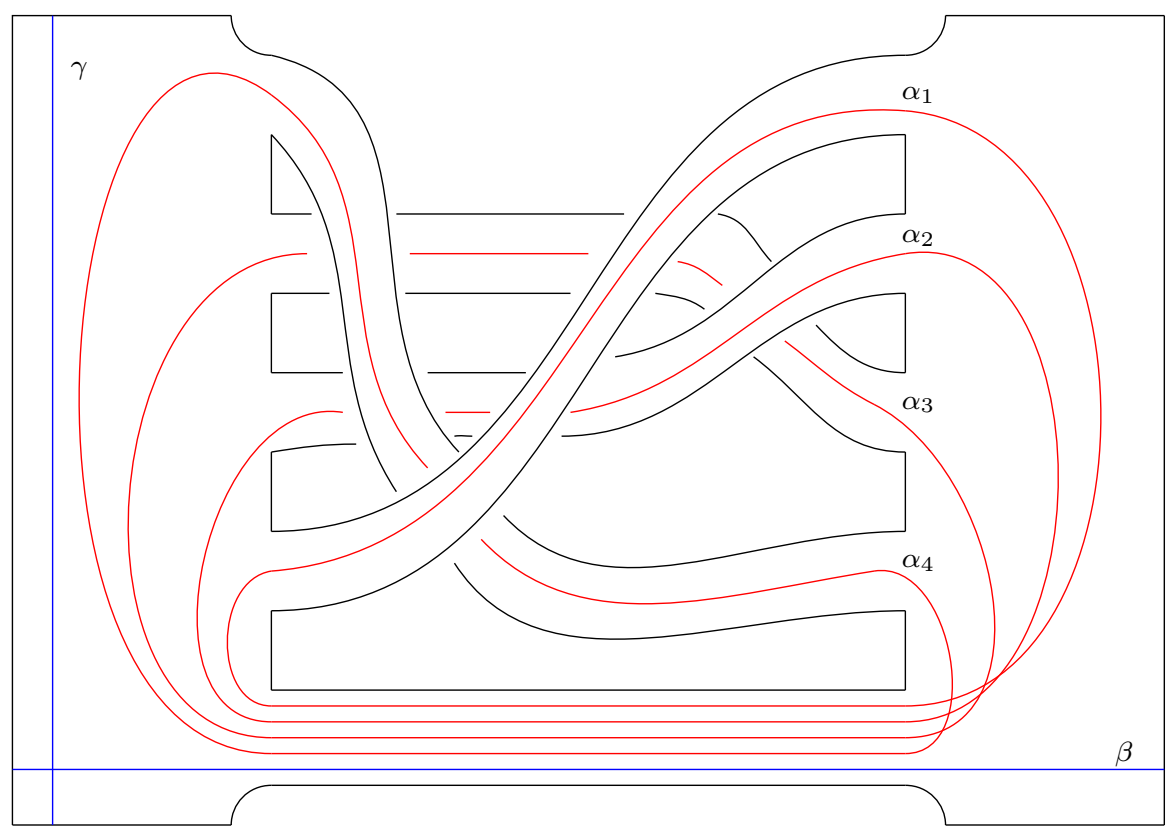

Fig. 9 Circular gluing with $r=5, k=1$ (left-right, top-bottom are identified)

Next, we observe that the winding numbers along either $\beta$ and $\gamma$ is zero, so $q_{\eta}(\beta)=$ $q_{\eta}(\gamma)=2$, and hence,

$$
\left(q_{\eta} / 2\right)(x \beta+y \gamma)=x^{2}+y^{2}+x y \bmod (2) .
$$

This immediately implies (i) and (ii) in the case of $\Sigma^{c i r}(r ; k)$. In the case of a general surface $\Sigma^{c i r}\left(r_{1}, \ldots, r_{n} ; k_{1}, \ldots, k_{n}\right)$, the space $H_{1}\left(\Sigma, \mathbb{Z}_{2}\right)$ is spanned by the classes of loops $\left(\alpha_{i, j}\right)_{j=1, \ldots, r_{i}-1}$ connecting $i$ th and $(i+1)$ st annuli (defined in the same way as $\left.\left(\alpha_{j}\right)\right)$, as well as by the classes of vertical loops $\gamma_{i}$, one in each annulus, and by the horizontal loop $\beta$. The restriction of $q_{\eta} / 2$ to the set of classes $\left(\alpha_{i, j}\right)$, for fixed $i$, agrees with the form $q\left(r_{i}, k_{i}\right)$ on $V\left(\sigma_{r_{i}}\left(k_{i}\right)\right)$. Furthermore, all the classes $\gamma_{i}-\gamma_{j}$ lie in the kernel of the intersection form, and the classes $\gamma_{i}$ and $\beta$ are orthogonal to $\left(\alpha_{i, j}\right)$. It follows that the quotient of $H_{1}\left(\Sigma, \mathbb{Z}_{2}\right)$ by the kernel of the intersection form splits into a direct sum of $\bar{V}\left(\sigma_{r_{i}}\left(k_{i}\right)\right)$ over $i=1, \ldots, n$, and the 2-dimensional subspace generated by the classes of the loops $\beta, \gamma=\gamma_{1}$. Now the result follows as in the case $n=1$.

We have the following analog of Theorem 4.2 for the circular gluing.

Theorem 4.8 The graded surface $\Sigma^{\text {cir }}\left(r_{1}, \ldots, r_{n} ; k_{1}, \ldots, k_{n}\right)$ is determined up to a graded homeomorphism by the unordered collection of numbers

$$
\left(\left(r_{1} / p_{1}\right)^{p_{1}}, \ldots,\left(r_{n} / p_{n}\right)^{p_{n}}\right)
$$


and in addition, in the case when all $r_{i} / p_{i}$ are odd, by the invariant $A(\eta)$ given by (4.3). Hence, the same data determines the category $D^{b}\left(\operatorname{Coh} R\left(r_{1}, \ldots, r_{n} ; k_{1}, \ldots, k_{n}\right)\right)$ up to equivalence.

Proof As before, this follows from Corollary 1.10. In the case when the genus is $\geq 2$, we know that the invariant $\sigma$ vanishes for our line field $\eta$, and the assertion follows from Lemma 4.7.

The case of genus 1 appears only when $k_{i}=-1$ for all $i$, in which case one immediately verifies that $\widetilde{A}(\eta)=0$.

\subsubsection{Case of irreducible stacky curves}

Assume that $n=1$. Using Theorem 4.8 we can find examples of different $k$ and $k^{\prime}$ such that the surfaces $\Sigma^{c i r}(r ; k)$ and $\Sigma^{c i r}\left(r ; k^{\prime}\right)$ are graded homeomorphic, so we get interesting examples of derived equivalences between irreducible stacky curves.

Corollary 4.9 Assume that $r \equiv 0 \bmod (4)$, and $k, k^{\prime} \in \mathbb{Z}_{r}^{*}$ are such that $k \equiv 1 \bmod (4)$, $k^{\prime} \equiv 1 \bmod (4)$, and $\operatorname{gcd}(k+1, r)=\operatorname{gcd}\left(k^{\prime}+1, r\right)$. Then we have an equivalence

$$
D^{b}(\operatorname{Coh} R(r ; k)) \simeq D^{b}\left(\operatorname{Coh} R\left(r ; k^{\prime}\right)\right)
$$

Proof In this case $k+1 \equiv 2 \bmod (4)$, so $p=\operatorname{gcd}(k+1, r) \equiv 2 \bmod (4)$ and hence, $r / p$ is even. It follows that the winding numbers of boundary components are divisible by 4 , so the Arf-invariant does not appear.

Now let us consider the case when $k \in \mathbb{Z}_{r}^{*}$ satisfies $\operatorname{gcd}(k+1, r)=1$. Note that this is possible only when $r$ is odd, and by Theorem 4.8 , the graded surface $\Sigma^{c i r}(r ; k)$ (that has genus $g=(r+1) / 2)$ depends on $k$ only through the Arf-invariant $A(\bar{q}(r, k))$.

We will compute this Arf-invariant for $k=1$ and $k=2$ in Sect. 4.3 below. By Theorem 4.8, this leads to the following derived equivalence.

Corollary 4.10 Assume that $r \geq 7$ is not divisible by 3 and $r \equiv \pm 1 \bmod (8)$. Then we have an equivalence

$$
D^{b}(\operatorname{Coh} R(r ; 1)) \simeq D^{b}(\operatorname{Coh} R(r ; 2)) .
$$

Proof By Lemma 4.14 below, for odd $r$, we have

$$
A(\bar{q}(r, 1))=\left(\begin{array}{c}
(r-1) / 2 \\
2
\end{array}\right) \bmod 2 .
$$

On the other hand, by Lemma 4.15, we have

$$
A(\bar{q}(r, 2))=(r-1) / 2 \bmod 2
$$

One can easily check that these two invariants are the same precisely when $r \equiv$ $\pm 1 \bmod (8)$. 


\subsubsection{Merging stacky nodes}

Note that the pairs $\left(r_{i}, k_{i}\right)$ with $k_{i}=-1$ do not contribute to the Arf-invariant $A(\eta)$ since in this case $\bar{V}\left(\sigma_{r_{i}}(-1)\right)=0$. Thus, the analog of Corollary 4.3 still holds.

Corollary 4.11 Let $I \subset[1, n-1]$ is a subset such that $k_{i}=-1$ for $i \in I$, and let $r_{I}=\sum_{i \in I} r_{i}$. Then there is an equivalence

$$
D^{b}\left(\operatorname{Coh} R\left(r_{1}, \ldots, r_{n} ; k_{1}, \ldots, k_{n}\right)\right) \simeq D^{b}\left(\operatorname{Coh} R\left(r_{I},\left(r_{i}\right)_{i \notin I} ;-1,\left(k_{i}\right)_{i \notin I}\right)\right)
$$

One has to be more careful with finding an analog of Corollary 4.4 since sometimes one has to compare the Arf-invariants. However, if some other winding numbers are divisible by 4 then the Arf-invariant does not appear.

Corollary 4.12 Assume that for $k \in \mathbb{Z}_{r}^{*}$ one has $\operatorname{gcd}(k+1, r)=1$, and let $d$ be a divisor of $k+1$. Assume also that there exists $i>d$ such that $r_{i} / d_{i}$ is even. Then we have an equivalence

$$
\begin{aligned}
& D^{b}\left(\operatorname{Coh} R\left((r)^{d}, r_{d+1}, \ldots, r_{n} ;(k)^{d}, k_{d+1}, \ldots, k_{n}\right)\right) \\
& \simeq D^{b}\left(\operatorname{Coh} R\left(d r, r_{d+1}, \ldots, r_{n} ; k, k_{d+1}, \ldots, k_{n}\right)\right) .
\end{aligned}
$$

Now let us consider an example where Arf-invariant does appear. Namely, for odd $r$, let us consider merging of two stacky nodes of type $(r ; 1)$ into one stacky node of type $(2 r ; 1)$. It turns out that the corresponding surfaces are homeomorphic but not necessarily graded homeomorphic.

Corollary 4.13 For odd $r$, there exists a graded homeomorphism between $\Sigma^{c i r}(r, r ; 1,1)$ and $\Sigma^{c i r}(2 r ; 1)$ if and only if $r \equiv 1 \bmod (4)$. Hence, for $r \equiv 1 \bmod (4)$, we have an equivalence

$$
D^{b}(\operatorname{Coh} R(r, r ; 1,1)) \equiv D^{b}(\operatorname{Coh} R(2 r ; 1))
$$

Proof For the graded surface $\Sigma^{c i r}(r, r ; 1,1)$, we have

$$
A(\eta)=2 A(\bar{q}(r, 1))+1=1 \bmod (2) .
$$

On the other hand, for $\Sigma^{c i r}(2 r ; 1)$, we have

$$
A(\eta)=A\left(\bar{q}(2 r, 1)+1=\frac{r+1}{2} \bmod (2)\right.
$$

by Lemma 4.14. Thus, the two Arf-invariants match exactly when $r \equiv 1 \bmod (4)$. 


\subsection{Computation of the Arf-invariants}

\section{Lemma 4.14 For odd $r$ one has}

$$
\begin{aligned}
& A(\bar{q}(r, 1))=\left(\begin{array}{c}
(r-1) / 2 \\
2
\end{array}\right) \bmod (2), \\
& A\left(\bar{q}(2 r, 1)=\frac{r-1}{2} \bmod (2) .\right.
\end{aligned}
$$

Proof Since $\sigma_{r}(1)$ is the order reversing permutation of $\{1, \ldots, r-1\}, q=q(r, 1)=$ $\bar{q}(r, 1)$ is the unique quadratic form on the $\mathbb{Z}_{2}$-vector space $V=V\left(\sigma_{r}(1)\right)$ with the basis $\alpha_{1}, \ldots, \alpha_{r-1}$, compatible with the symplectic pairing given by

$$
\alpha_{i} \cdot \alpha_{j}=1 \text { for } i \neq j,
$$

and satisfying $q\left(\alpha_{i}\right)=0$ for all $i$.

It is well known that the Gauss sum

$$
G(q):=\sum_{x \in V}(-1)^{q(x)}
$$

is equal to $\pm 2^{(r-1) / 2}$ and its sign determines the Arf-invariant. It is easy to see that for any $x \in V$, one has

$$
q(x)=(-1)^{\left(\begin{array}{l}
k \\
2
\end{array}\right)},
$$

where $k$ is the number of nonzero coordinates of $x$. Thus, we have

$$
G(q)=\sum_{k=0}^{r-1}\left(\begin{array}{c}
r-1 \\
k
\end{array}\right)(-1)^{\left(\begin{array}{l}
k \\
2
\end{array}\right) .}
$$

Now we observe that

$$
(-1)^{\left(\begin{array}{c}
k \\
2
\end{array}\right)}=\frac{1-i}{2} \cdot i^{k}+\frac{1+i}{2} \cdot(-i)^{k},
$$

where $i=\sqrt{-1}$. Thus, we have

$$
\begin{aligned}
& \sum_{k=0}^{r-1}\left(\begin{array}{c}
r-1 \\
k
\end{array}\right)(-1)^{\left(\begin{array}{l}
k \\
2
\end{array}\right)}=\frac{1-i}{2} \cdot(1+i)^{r-1}+\frac{1+i}{2} \cdot(1-i)^{r-1} \\
& \quad=2^{(r-1) / 2} \cdot\left[\frac{1-i}{2} \cdot i^{(r-1) / 2}+\frac{1+i}{2} \cdot(-i)^{(r-1) / 2}\right]=2^{(r-1) / 2} \cdot(-1)^{\left(\begin{array}{c}
(r-1) / 2 \\
2
\end{array}\right)},
\end{aligned}
$$

which proves our formula for the Arf-invariant of $\bar{q}(r, 1)$. 
Now, let us consider the form $q=q(2 r, 1)$ on the vector space $W=V\left(\sigma_{2 r}(1)\right)$ with the basis $\alpha_{1}, \ldots, \alpha_{2 r-1}$, equipped with the even pairing given by (4.4), where $q$ is compatible with the pairing and satisfies $q\left(\alpha_{i}\right)=0$. Note that the kernel of the pairing is spanned by the vector $v_{0}=\sum_{k=1}^{2 r-1} \alpha_{k}$, and we have

$$
q\left(v_{0}\right)=\left(\begin{array}{c}
2 r-1 \\
2
\end{array}\right)=0 \bmod (2),
$$

since $r$ is odd. Thus, the form $q$ descends to a nondegenerate quadratic form $\bar{q}=$ $\bar{q}(2 r, 1)$ on $\bar{W}=W /\left\langle v_{0}\right\rangle$. We claim that its Arf-invariant is

$$
A(\bar{q})=\frac{r-1}{2} \bmod 2 .
$$

Indeed, again we consider the Gauss sum

$$
G(\bar{q}):=\sum_{x \in \bar{W}}(-1)^{\bar{q}(x)} .
$$

We have

$$
\begin{aligned}
G(\bar{q}) & =\frac{1}{2} \cdot G(q)=\frac{1}{2} \sum_{k=0}^{2 r-1}\left(\begin{array}{c}
2 r-1 \\
k
\end{array}\right)(-1)^{\left(\begin{array}{c}
k \\
2
\end{array}\right)} \\
& =\frac{1-i}{4} \cdot(1+i)^{2 r-1}+\frac{1+i}{4}(1-i)^{2 r-1}=(-4)^{(r-1) / 2}
\end{aligned}
$$

Lemma 4.15 Assume that $r$ is odd and not divisible by 3. Then

$$
A(\bar{q}(r, 2))=\frac{r-1}{2} \bmod (2) .
$$

Proof The form $q=q(r, 2)=\bar{q}(r, 2)$ is in $\operatorname{Quad}(V)$, where $V$ is the $\mathbb{Z}_{2}$-space with the basis $\alpha_{1}, \ldots, \alpha_{r-1}$ and the symplectic pairing given by

$$
\alpha_{i} \cdot \alpha_{j}= \begin{cases}0, & i<j<i+(r-1) / 2 \\ 1, & \text { otherwise }\end{cases}
$$

where $i<j$. Furthermore, $q$ is determined by $q\left(\alpha_{i}\right)=0$ for all $i$. It is easy to see that by renumbering the classes $\left(\alpha_{i}\right)$ as follows:

$$
\alpha_{1}^{\prime}=\alpha_{(r-1) / 2}, \ldots, \alpha_{(r-1) / 2}^{\prime}=\alpha_{1}, \alpha_{(r-1) / 2+1}^{\prime}=\alpha_{r-1}, \ldots, \alpha_{r-1}^{\prime}=\alpha_{(r-1) / 2+1}^{\prime},
$$


we get

$$
\alpha_{i}^{\prime} \cdot \alpha_{j}^{\prime}= \begin{cases}1, & i<j<i+(r-1) / 2 \\ 0, & j \geq i+(r-1) / 2\end{cases}
$$

and $q \in \operatorname{Quad}(V)$ still satisfies $q\left(\alpha_{i}^{\prime}\right)=0$ for all $i$.

We will compute the Arf-invariant by relating $(V, q)$ to another space with a quadratic form. For every $k \geq 0$, such that $k \not \equiv 2 \bmod (3)$, let us consider a $\mathbb{Z}_{2}$-vector space $W_{k}$ with the basis $\beta_{1}, \gamma_{1}, \ldots, \beta_{k}, \gamma_{k}$, the even pairing given by the rule

$$
\begin{aligned}
& \beta_{i} \cdot \beta_{j}=1 \text { for } i \neq j ; \quad \gamma_{i} \cdot \gamma_{j}=1 \text { for } i \neq j \\
& \beta_{i} \cdot \gamma_{j}=1 \text { for } i \leq j ;
\end{aligned}
$$

and the quadratic form $q_{k}$ in $\operatorname{Quad}\left(W_{k}\right)$ such that $q_{k}\left(\beta_{i}\right)=q\left(\gamma_{i}\right)=1$ for all $i$.

First, we will prove that $A(q)=A\left(q_{(r-1) / 2-2}\right)$ and then we will prove that

$$
A\left(q_{k}\right)=k \bmod (2) \text {. }
$$

To relate $(V, q)$ with $\left(W_{(r-1) / 2-2}, q_{(r-1) / 2-2}\right)$ let us consider the 2-dimensional isotropic subspace $I \subset V$ spanned by $\alpha_{1}^{\prime}$ and $\alpha_{r-1}^{\prime}$. We have $\left.q\right|_{I} \equiv 0$, so the Arfinvariant of $q$ is equal to that of the induced quadratic form on $I^{\perp} / I$. Now setting

$$
\gamma_{i}=\alpha_{2}^{\prime}+\alpha_{2+i}^{\prime}, \quad \beta_{i}=\alpha_{(r-1) / 2+1}^{\prime}+\alpha_{(r-1) / 2+1+i},
$$

for $i=1, \ldots,(r-1) / 2-2$, we get an identification of $I^{\perp} / I$ with $W_{(r-1) / 2-2}$, compatible with the quadratic forms. Hence, $A(q)=A(q(r-1) / 2-2)$.

To prove (4.5) we use induction on $k$. It is easy to check that $A\left(q_{1}\right)=1$ (and $A\left(q_{0}\right)=0$ for trivial reasons), so it is enough to establish the formula

$$
A\left(q_{k}\right)=A\left(q_{k-3}\right)+1 \text {. }
$$

To this end we consider the 2-dimensional isotropic subspace $J \subset W_{k}$ spanned by $\beta_{k}+\gamma_{1}$ and $\beta_{1}+\beta_{k}+\gamma_{k}$. We have $\left.q_{k}\right|_{J}=0$, and our formula follows from the identification

$$
J^{\perp} / J \simeq W_{k-3} \oplus W_{1},
$$

where the standard basis of $W_{k-3}$ corresponds to the elements

$$
\left(\beta_{2}+\beta_{2+i} \bmod J, \gamma_{2}+\gamma_{2+i} \bmod J\right)_{1 \leq i \leq k-3}
$$

while a copy of $W_{1}$ spanned by $\beta_{k} \bmod J$ and $\gamma_{k} \bmod J$. 
Acknowledgements Y.L. is partially supported by the Royal Society (URF) and the NSF grant DMS1509141, and would like to thank Martin Kalck for pointing out the reference [7]. A.P. is supported in part by the NSF grant DMS-1700642 and by the Russian Academic Excellence Project '5-100'. While working on this project, A.P. was visiting King's College London, Institut des Hautes Etudes Scientifiques, and Korea Institute for Advanced Study. He would like to thank these institutions for hospitality and excellent working conditions.

Open Access This article is distributed under the terms of the Creative Commons Attribution 4.0 International License (http://creativecommons.org/licenses/by/4.0/), which permits unrestricted use, distribution, and reproduction in any medium, provided you give appropriate credit to the original author(s) and the source, provide a link to the Creative Commons license, and indicate if changes were made.

\section{References}

1. Amiot, C.: The derived category of surface algebras: the case of the torus with one boundary component. Algebras Represent. Theory 19(5), 1059-1080 (2016)

2. Arf, C.: Untersuchungen über quadratische Formen in Körpern der Charakteristik 2. I. J. Reine Angew. Math. 183, 148-167 (1941)

3. Assem, I., Skowrónski, A.: Iterated tilted algebras of type $\tilde{A}_{n}$. Math. Z. 195(2), 269-290 (1987)

4. Atiyah, M.: Riemann surfaces and spin structures. Ann. Sci. École Norm. Sup. 4, 47-62 (1971)

5. Auroux, D.: Fukaya categories of symmetric products and bordered Heegaard-Floer homology. J. Gökova Geom. Topool

6. Auroux, D., Katzarkov, L., Orlov, D.: Mirror symmetry for weighted projective planes and their noncommutative deformations. Ann. Math. (2) 167(3), 867-943 (2008)

7. Avella-Alaminos, D., Geiss, C.: Combinatorial derived invariants for gentle algebras. J. Pure Appl. Algebra 212(1), 228-243 (2008)

8. Bardzell, M.J.: The alternating syzygy behavior of monomial algebras. J. Algebra 188, 69-89 (1997)

9. Bessenrodt, C., Holm, T.: Weighted locally gentle quivers and Cartan matrices. J. Pure Appl. Algebra 212(1), 204-221 (2008)

10. Bocklandt, R.: Noncommutative mirror symmetry for punctured surfaces, with an appendix by Mohammed Abouzaid. Trans. Am. Math. Soc. 368(1), 429-469 (2016)

11. Burban I., Drozd Y.: On the derived categories of gentle and skew-gentle algebras: homological algebra and matrix problems, preprint arXiv:1706.08358

12. Dickson, L.E.: Linear Groups with an Exposition of the Galois Field Theory. Cosimo Classics, New York (2007)

13. Ekholm, T., Lekili, Y.: Duality between Lagrangian and Legendrian invariants, preprint arXiv: 1701.01284

14. Fock, V.V., Rosly, A.A.: Poisson structure on moduli of flat connections on Riemann surfaces and the r-matrix. In: Moscow Seminar in Mathematical Physics, 67-86, Amer. Math. Soc. Transl. Ser. 2, 191, Adv. Math. Sci., 43, Amer. Math. Soc., Providence, RI (1999)

15. Haiden, F., Katzarkov, L., Kontsevich, M.: Flat surfaces and stability structures. Publ. Math. Inst. Hautes Études Sci. 126, 247-318 (2017)

16. Hopf, H.: Differential Geometry in the Large, Lecture Notes in Mathematics 1000. Springer, Berlin (1983)

17. Johnson, D.: Spin structures and quadratic forms on surfaces. J. Lond. Math. Soc. (2) 22(2), 365-373 (1980)

18. Kawazumi, N.: The mapping class group orbits in the framings of compact surface, preprint arXiv: 1703.02258

19. Keller, B.: Deriving DG categories. Ann. Sci. École Norm. Sup. (4) 27(1), 63-102 (1994)

20. Kontsevich, M.: Homological algebra of mirror symmetry, Proceedings of the International Congress of Mathematicians, Vol. 1, 2 (Zürich, 1994), 120-139 (1994)

21. Lekili, Y., Polishchuk A.: Auslander orders over nodal stacky curves and partially wrapped Fukaya categories. J. Topology 11, 615-644 (2018)

22. Nadler, D., Zaslow, E.: Constructible sheaves and the Fukaya category. J. Am. Math. Soc. 22(1), 233-286 (2009) 
23. Opper S., Plamondon, P.-G., Schroll S.: A geometric model for the derived category of gentle algebras, preprint arXiv: 1801.09659

24. Polishchuk A., Van den Bergh M.: Semiorthogonal decompositions of the categories of equivariant coherent sheaves for some reflection groups. JEMS 21, 2653-2749 (2019)

25. Ringel, C.M.: The repetitive algebra of a gentle algebra. Bol. Soc. Mat. Mexicana 3(2), 235-253 (1997)

26. Seidel, P.: Graded Lagrangian submanifolds. Bull. Soc. Math. Fr. 128(1), 103-149 (2000)

27. Seidel, P.: Fukaya Categories and Picard-Lefschetz Theory. EMS, Zürich (2008)

28. Sibilla, N.: Mirror Symmetry in Dimension 1 and Fourier-Mukai Equivalences. In: Homological Mirror Symmetry and Tropical Geometry, pp. 407-428. Springer, Cham (2014)

29. Sibilla, N., Treumann, D., Zaslow, E.: Ribbon graphs and mirror symmetry. Sel. Math. (N.S.) 20(4), 979-1002 (2014)

Publisher's Note Springer Nature remains neutral with regard to jurisdictional claims in published maps and institutional affiliations. 